\title{
A Traffic Assignment Model Based on Link Densities
}

\author{
Louis de Grange $\mathbb{D}^{1},{ }^{1}$ Matthieu Marechal, ${ }^{2}$ and Felipe González ${ }^{1}$ \\ ${ }^{1}$ School of Industrial Engineering, Diego Portales University, Santiago de Chile, Chile \\ ${ }^{2}$ Institute of Basic Sciences, Faculty of Engineering, Diego Portales University, Santiago de Chile, Chile
}

Correspondence should be addressed to Louis de Grange; louis.degrange@udp.cl

Received 31 December 2018; Revised 23 April 2019; Accepted 2 June 2019; Published 27 June 2019

Academic Editor: Francesco Viti

Copyright (c) 2019 Louis de Grange et al. This is an open access article distributed under the Creative Commons Attribution License, which permits unrestricted use, distribution, and reproduction in any medium, provided the original work is properly cited.

\begin{abstract}
A new model is presented that determines the traffic equilibrium on congested networks using link densities as well as costs in a manner consistent with the fundamental traffic equation. The solution so derived satisfies Wardrop's first principle. This densitybased approach recognizes traffic flow reductions that may occur when network traffic congestion is high; also, it estimates queue lengths (i.e., the number of vehicles on saturated links), and it explicitly takes into account the maximum flow a link can handle, which is defined by the fundamental traffic equation. The model is validated using traffic microsimulations and implemented on a typical Nguyen-Dupuis network to compare it with a flow-based approach. System optimal assignment model based on link densities is also presented.
\end{abstract}

\section{Introduction}

This paper develops and implements a deterministic model that solves the traffic equilibrium problem for a congested road network using network link densities. More specifically, the proposed density-based model solves a variational inequality whose cost vector is a function of the number of vehicles seeking to travel on the network at a given instant, consistent with the relationship between flow, cost (the inverse of speed), and density given by the fundamental traffic equation (flow $=$ speed $\times$ density) for each network link. The solution arrived at gives a network traffic equilibrium that satisfies Wardrop's first principle [1] and whose costs depend on link densities.

Using the link densities to obtain the traffic equilibrium on a congested network has several important advantages. In general terms, the modelling of the problem is more realistic than that achieved by the classical flow-based traffic assignment formulations employing monotonically increasing cost functions. More specifically, the density-based approach has the following desirable features.

(i) It recognizes that link capacity (or maximum flow) is neither fixed nor exogenous but rather depends on the density level. In other words, the maximum flow that can cross a link varies as a function of link density. This prevents the flow from exceeding the link's physical capacity. The capacities or maximum flows of the links in a network are determined by each link's traffic speed and density as determined by the fundamental traffic equation.

(ii) It determines whether a reduced flow level on a given link is due to low latent demand for its use (e.g., low density) or rather to the presence of traffic congestion (e.g., high density) limiting the amount of flow able to use the link and generating queues and longer delays.

(iii) The average queue length on each link can be estimated.

(iv) By generating estimates of the impact of densities on the flow levels that can circulate on network links, the approach provides important data for use in the design of road networks, highway entrance and exit ramps, and road pricing systems based on traffic saturation levels. This is a distinct advantage over flow-based models, which estimate only flows.

However, the density-based approach has a disadvantage: to find a solution which satisfies the flow conservation along the network is, in general, complicated. The implementation of numerical methods which allows for solutions which satisfy 
the flow conservation to be found will be the topic of future works.

The remainder of this paper is divided into four sections and three appendices. Section 2 contains a brief review of the literature on traffic assignment models, emphasizing the limits of classical deterministic flow-based models that assume a monotonically increasing relationship between flow and cost on each network link. Section 3 introduces our density-based traffic equilibrium model satisfying Wardrop's first principle and discusses the existence of an equilibrium solution. This section also suggests an analytic expression for estimating the benefits of a road system project or policy using the proposed model in terms of consumer surplus before and after implementation. Section 4 uses a numerical example to compare our density-based model with a flowbased model that assumes a monotonically increasing relationship between link flows and costs. Section 5 summarizes our main conclusions.

Appendix A validates the proposed model using microsimulations of a small road network; Appendix B gives a more formal demonstration in support of the existence theorems given in Section 3; and finally, Appendix $\mathrm{C}$ presents the system optimal assignment model based on link densities, which will allow us to advance in future new lines of research.

\section{Literature Review}

A widely accepted result in the study of vehicles on congested road networks is the so-called Wardrop equilibrium, also known as Wardrop's first principle of route choice [1]. It is equivalent to the concept put forward by Knight [2] as a simple behavioural rule for describing the way trips are distributed across alternative routes under congested conditions.

Wardrop's first principle states that the vehicle travel time or cost for every network route used will be equal to or less than the time or cost that would be experienced on any unused route. Each user attempts to minimize noncooperatively their trip cost or time. Traffic flows that satisfy this principle are generally referred to as "user equilibrium" (UE) flows since each user chooses the route they find best. In short, a user-optimal equilibrium is reached when no user can reduce their travel time or cost on the network through unilateral action.

The first mathematical model of user equilibrium on a congested road network assuming a monotonically increasing relationship between the flows and costs along the network links was formulated by Beckmann et al. [3], using a nonlinear optimization problem. However, traffic assignment problems based on link flows have generally been addressed using variational inequality, to include asymmetric cost functions, or multivariable cost functions. Notable among works that treat the formulation of the equilibrium problem, the existence of a solution and the solution algorithm are the following: Dafermos and Sparrow [4], Smith [5], Dafermos $[6,7]$, Florian and Spiess [8], Fisk and Nguyen [9], Fisk and Boyce [10], Nagurney [11], Hammond [12], Marcotte and Guelatt [13], Auchmuty [14], Gabriel and Bernstein [15], and
Patriksson [16]. All these works represent mainly theoretical contributions and algorithmic implementation.

A variation on Wardrop is the stochastic user equilibrium (SUE), in which no user can unilaterally change routes to improve their perceived travel cost or time. Some stochastic or probabilistic approaches are used, under a similar theoretical framework, to represent different phenomena, such as uncertainty, randomness, and/or heterogeneity of users and route alternatives. The precise formulation depends on how these factors are incorporated. Surveys of this class of models are found in Daganzo and Sheffi [17], Hazelton [18], Ramming [19], Prashker and Bekhor [20], Karoonsoontawong and Lin [21], Li and Huang [22], and Batista et al. [23]. These formulations constitute an extension of the deterministic equilibrium models. Lim and Kim [24] combine trip distribution and route choice model into a single feedback process within a single framework.

Both UE and SUE models typically assume a monotonically increasing relationship between cost and flow (see $[25,26])$. This ensures that they are practical to implement and their results are easy to analyze and interpret, but for heavily or hypercongested situations they are greatly lacking in realism.

Another major limitation of flow-based approaches is that they allow the assignment of flow levels that exceed link capacity. Notice that Beckmann model does not specify cost or capacity functions, but it does assume that these functions must be monotonous and growing. Depending on the cost function considered for network links, the flow may exceed the defined capacity, for example, with increasing monotonic functions [27]. This unrealistic result has been interpreted as the additional wait time (or queuing time to enter the network), but the main problem is that it overestimates the number of vehicles which can circulate on the network's links in a given time period.

A third important drawback with flow-based approaches is that they assume link maximum flows are fixed, exogenous parameters, yet, as the fundamental traffic equation indicates, maximum link flow depends on density which in turn is related to the demand for link use. In other words, link maximum flow is more like a variable than a fixed parameter.

It is precisely these various shortcomings that are remedied by our density-based model, set out below in Section 3 .

Note that extensions to flow-based assignment models incorporating an additional restriction barring each link's flow from exceeding a fixed and exogenous capacity have been developed by Larsson and Patriksson [28], Ferrari [29], and Nie et al. [30]. These modifications do avoid the problem of physically overloaded links, but they still assume a monotonically increasing flow-delay relationship that, as already mentioned, is unrealistic when congestion is high.

Dynamic assignment models have been comprehensively studied in the specialized literature but are not directly related to the approach we propose here. Ran and Boyce [31], Peeta and Ziliakopoulus [32], and Tampere and Viti [33] provide extensive references and discuss developments in dynamic transportation network modelling and analysis and associated computational methods. Surveys have also been 
published by Boyce et al. [34] and Szeto and Lo [35] and, more recently, by Liu et al [36].

Another approach that better captures the flow-delay relationship (which is increasing under low congestion and decreasing under high congestion) in the fundamental traffic equation uses traffic microsimulation models. A recent survey on the state of the art in traffic assignment models using microsimulation may be found in Calvert et al. [37]. These tools allow physical road network restrictions and the effects of density on traffic flow and speed to be incorporated into the modelling, but they are difficult to apply to large networks. Furthermore, microsimulation models do not use the traffic equilibrium concept since by definition they are dynamic formulations for individual behaviour that base route choice rather on heuristics.

For this reason, microsimulation is an approach that allows us to validate our new model, as we explain in Appendix A. Microsimulation considers each of the vehicles moving within the network as the unit of analysis. Each vehicle makes decisions based on its destination, the behavior of other vehicles, and the travel times perceived between alternative routes that are the result of vehicle interactions. Flow-delay functions in the links are not required; however, these flow-delay functions can be estimated as a result of the individual interactions of the vehicles traveling in the different arcs of the microsimulated network. These interactions include the effect of density on traffic circulation, crossing blocks, etc. Therefore, microsimulation is an approach that we can consider as a benchmark for our new model in small networks. In larger networks, with many vehicles circulating simultaneously, microsimulation has limitations, especially due to the increase in alternative routes, or convergence of results. It is in these cases where we consider that our new model presents the greatest advantages.

\section{Formulation of the Proposed Traffic Assignment Model}

3.1. General Definitions. Traffic flows typically are not uniform but rather vary across space and time, making them difficult to describe. Nevertheless, their behaviour has traditionally been explained in terms of the relationships between just three traffic variables: flow, speed, and density (the lattermost also known as concentration).

In a deterministic approach, mean speed $(V)$ is defined as the average speed of multiple vehicles crossing a specific point or link. The flow or volume $(f)$ is defined as the number of vehicles crossing a given road or highway segment during a given period of time. Finally, density $(D)$ is defined as the number of vehicles occupying that segment.

For roads or highways with multiple lanes, flow is expressed as vehicles per time unit per number of lanes [38]. The same is true of density, which is also expressed in terms of the total width of the route. A good survey of these models may be consulted in Wang et al. [39] and Kucharski and Drabicki [40].

The oldest and probably simplest macroscopic traffic flow model was proposed by Greenshield (1935). It assumes that, under uninterrupted flow conditions, speed and density are linearly related. Although Greenshield's formulation is considered to be the tool with the widest scope for traffic flow modelling due to its simplicity and reasonable goodnessof-fit, it has not been universally accepted given that it does not provide a good fit when congestion is low. The formal expression of the Greenshield model is as follows:

$$
\begin{aligned}
& V=v_{0} \cdot\left[1-\frac{D}{D_{\text {jam }}}\right] \longrightarrow \\
& V=\alpha-\beta D, \\
& \alpha=v_{0}, \\
& \beta=\frac{1}{D_{\text {jam }}}
\end{aligned}
$$

where $V$ is the speed corresponding to the density level $D, v_{0}$ is the free-flow speed, and $D_{j a m}$ is the so-called jam density. This last term refers to the extreme traffic density level associated with completely stopped traffic flow, usually in the range of 185-250 vehicles per mile per lane. In other words, it is the density when flow is zero. A very recent study relating speed to vehicles' use of space in an equilibrium context is reported in Martínez-Díaz and Pérez [41]. There are other, more complex specifications of the relationship between speed and density, but for our purposes Equation (1) is sufficient.

From the fundamental traffic equation, we can relate the flow $\left(f_{a}\right)$ for a given network link $a$ with the flow's speed $\left(V_{a}\right)$ and density $\left(D_{a}\right)$ as $f_{a}=V_{a} \cdot D_{a}$. The speed can in turn be related with the link's cost or time $\left(c_{a}\right)$ and its length $\left(l_{a}\right)$ as $V_{a}=l_{a} / c_{a} \longrightarrow c_{a}=l_{a} / V_{a} \longrightarrow c_{a}\left(D_{a}\right)=l_{a} / V_{a}\left(D_{a}\right)$. Finally, if we define $N_{a}$ as the number of vehicles along $a$, then $N_{a}=$ $l_{a} \cdot D_{a}$.

An advantage of using the expression $V_{a}=V_{a}\left(D_{a}\right)$ is that it allows the cost of a link to be defined as a function of its density rather than its flow and can thereby capture the typical situation depicted in Figure 1 in which congestion results in a reversal of the flow-delay curve so that for a given flow level there may be two travel cost or time alternatives, such as $c_{a}^{*}$ and $c_{a}^{* *}$ in the figure. This relationship may be compared with the less realistic portrayal in Figure 2 that is typical of traffic equilibrium models incorporating congestion [3]. Clearly, the assumption in such models of a monotonically increasing flow-cost relationship limits their ability to accurately represent heavily congested conditions.

Another advantage of using density instead of flow is that it permanently incorporates a restriction on the maximum flow of the links or routes in the network. Such a restriction is illustrated in Figure 1, where flow cannot exceed $f_{a}^{\max }$. For each link $a$, this maximum is associated with a given density $D_{a}^{*}$. In other words, there exists a density $D_{a}^{*}$ at which the link's maximum flow, defined as $f_{a}^{\max }$, is obtained. As an example, assuming a typical relationship between speed and density such as that defined by Greenshield (1935) and De Grange et al. [42], in which $V_{a}\left(D_{a}\right)=\alpha_{a}-\beta_{a} D_{a}$, where $\alpha_{a}>0$ and $\beta_{a}>0$, it is easily shown that $D_{a}^{*}=\alpha_{a} / 2 \beta_{a}$ and $f_{a}^{\max }=\alpha_{a}^{2} / 4 \beta_{a}$. The maximum density a link can support, 


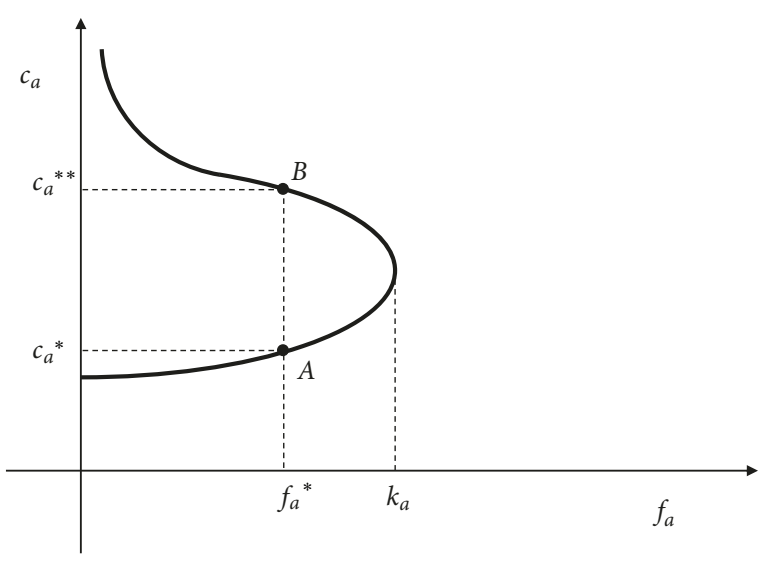

FIGURE 1: Relatively realistic flow-delay relationship on road link.

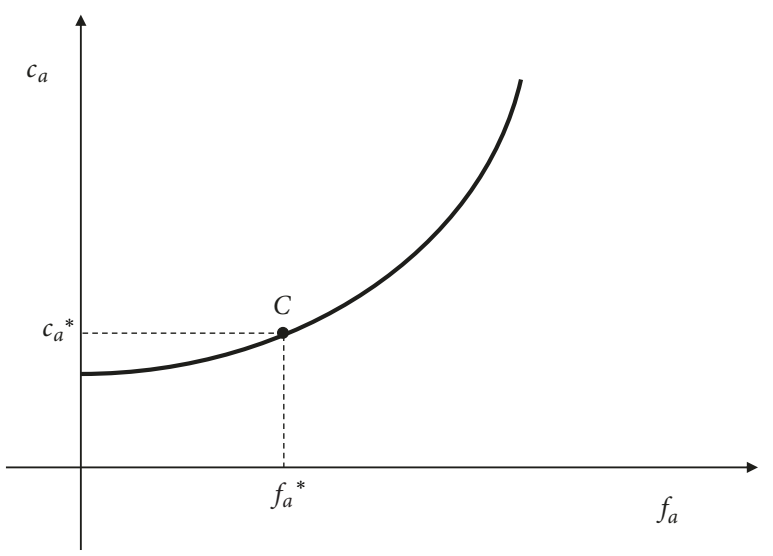

FIGURE 2: Relatively unrealistic flow-delay relationship on road link.

defined to be the density at which the speed across the link is 0 , is also directly derivable as $D_{a}^{\max }=\alpha_{a} / \beta_{a}$. Therefore, $0 \leq D_{a}<\alpha_{a} / \beta_{a}$.

Finally, the cost-density relationship is as shown in Figure 3. Note that substituting $N_{a}$ for $D_{a}$ would not change the properties illustrated in the figure.

\subsection{Analytic Formulation of Proposed Model Based on Link Densities}

3.2.1. Description of the Model. Let $W$ be the set of all origindestination $(\mathrm{O}-\mathrm{D})$ pairs, $P$ the set of all routes across the network, and $L$ the set of links. We define an incidence matrix $\Delta=\left(\delta_{a p}\right)_{a \in L, p \in P}$ such that $\delta_{a p}=1$ if $p$ passes through $a$; otherwise $\delta_{a p}=0$. For each route $p$ there exists a unique OD pair $w \in W$ such that $p$ joins $w$.

Let $N_{p}$ be the number of vehicles that travel on route $p$ and $N_{a}$ the number of vehicles present on link $a$. We assume that the quantities $N_{p}$ and $N_{a}$ are related by the following relation:

$$
N_{a}=\sum_{p \in P} q_{a p} N_{p}
$$

The quantities $q_{a p}=q_{a p}(N)$ satisfy the following:

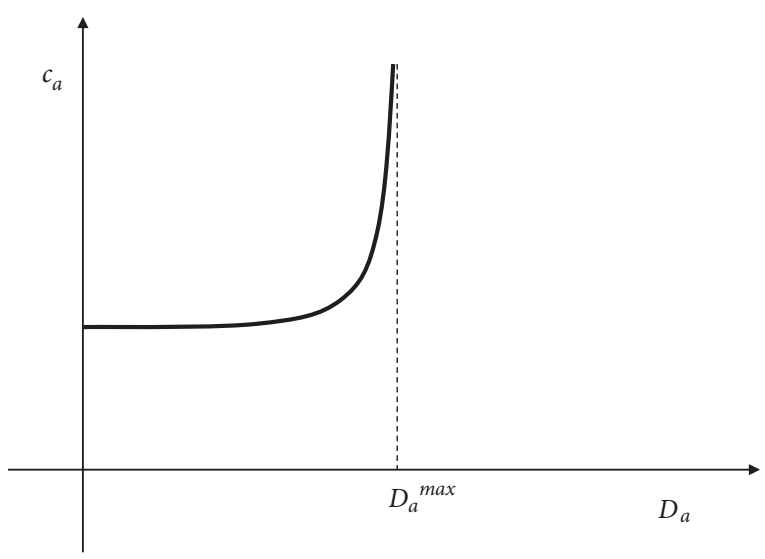

FIGURE 3: Density-delay relationship on road link.

(1) For all $a \in L$ and $p \in P, q_{a p}=0 \Longleftrightarrow \delta_{a p}=0$.

(2) For all $a \in L$ and $p \in P, q_{a p} \geq 0$.

(3) For all $p \in P, \sum_{a \in L} q_{a p}=1$.

It is important to observe that if $q_{a p}=\left(t_{a} / t_{p}\right) \delta_{a p}$, where $t_{a}$ is the time for travelling link $a$ and $t_{p}=\sum_{a} t_{a} \delta_{a p}$ is the time for travelling route $p$, then we have $N_{a} / t_{a}=\sum_{p \in P} \delta_{a p}\left(N_{p} / t_{p}\right)$. Let $f_{a}$ be the flow of vehicles on link $a$ and $h_{p}$ the flow of vehicles along route $p$; we have $N_{a} / t_{a}=f_{a}$ and $N_{p} / t_{p}=h_{p}$, which implies that $f_{a}=\sum_{p \in P} \delta_{a p} h_{p}$. Then the choice $q_{a p}=$ $\left(t_{a} / t_{p}\right) \delta_{a p}$ implies that flow conservation is satisfied.

Actually the travel time $t_{a}$ depends on the quantity of vehicles $N_{a}$ on link $a$ which makes the use of $t_{a}$ complicated.

An expression for $c_{a}$ is given by $t_{a}=l_{a} / v_{a}$ which is the time for travelling link $a$, with $l_{a}$ the length of link $a$ and $v_{a}$ the average speed of the vehicles along link $a$. A typical expression for $v_{a}$ is given by $v_{a}=\alpha_{a}-\beta_{a} D_{a}$, with $\alpha_{a}$ and $\beta_{a}$ two positive constants and $D_{a}=N_{a} / l_{a}$ the density of vehicles present on link $a$. Then we obtain

$$
c_{a}\left(N_{a}\right)=\frac{l_{a}}{\alpha_{a}-\beta_{a} D_{a}}=\frac{l_{a}}{\alpha_{a}-\beta_{a}\left(N_{a} / l_{a}\right)}
$$

Given a vector of the quantities of vehicles on routes denoted $N=\left(N_{p}\right)_{p \in P}$, we define the cost of each route $p$ as $c_{p}\left(N_{p}\right)=$ $\sum_{a \in L} c_{a}\left(N_{a}\right) \delta_{a p}$, where $c_{a}\left(N_{a}\right)$ is a positive, continuous, and increasing function on the interval $\left[0, N_{a}^{\max }\right)$. The quantity $N_{a}^{\max }$ is the maximum amount of vehicles that can be present on link $a$, and we have

$$
\lim _{N_{a} \longrightarrow N_{a}^{\max }} c_{a}\left(N_{a}\right)=+\infty
$$

Based on the foregoing definitions, we can now express Wardrop's first principle analytically as the conditions set out in (5) below, where $\lambda^{w}$ is the cost of travelling the routes actually used that connect O-D pair $w$, and $\mu_{p} \geq 0$ is 
the additional cost of travelling routes not used (the latter, according to the principle, having a higher cost). Consider

$$
\begin{aligned}
& \sum_{a \in L} c_{a}\left(N_{a}\right) \cdot \delta_{a p}=\lambda^{w}+\mu_{p}, \quad \forall w \in W, \forall p \in P^{w} \\
& N_{a}=\sum_{p \in P} N_{p} q_{a p} \quad \forall a \in L \\
& N^{w}=\sum_{p \in P^{w}} N_{p} \quad \forall w \in W \\
& N_{p} \geq 0 \quad \forall p \in P \\
& \mu_{p} \geq 0 \quad \forall p \in P \\
& N_{p} \mu_{p}=0 \quad \forall p \in P \\
& N_{a}<N_{a}^{\max } \quad \forall a \in L
\end{aligned}
$$

In the third equation $N^{w}$ is the quantity of vehicles that are travelling the origin-destination pair $w$ and $P^{w}$ is the set of routes joining the pair origin-destination $w$.

The solution to this system of equations gives the traffic equilibrium based on densities that we are seeking. Since we assume $c_{a}\left(N_{a}^{\max }\right)=+\infty$, the inequality relating $N_{a}$ with $N_{a}^{\max }$ is strict.

3.2.2. Existence of a Solution. To prove the existence of a solution to the system of equations set forth in (5) that is consistent with Wardrop's first principle, we adapt the system to the variational inequality format. To this end, we first define a set of feasibles $K$ as

$$
\begin{aligned}
K & =\left\{N=\left(N_{p}\right)_{p \in P} \mid N_{a}=\sum_{p \in P} N_{p} q_{a p}, N_{p} \geq 0, N_{a}\right. \\
& \left.<N_{a}^{\max }, N^{w}=\sum_{p \in P^{w}} N_{p}\right\}
\end{aligned}
$$

Finding an equilibrium solution consistent with Wardrop's first principle is equivalent to solving the following variational inequality, that is, finding a vector of quantities of vehicles on routes $N^{*} \in K$ such that, for all $N \in K$, we have $\sum_{p} c_{p}\left(N^{*}\right)\left(N_{p}-N_{p}^{*}\right) \geq 0$. Given the vector field $C=C(N)=$ $\left(C_{p}(N)\right)_{p \in P}$, the problem is to find a vector $N^{*} \in K$ such that, for all $N \in K$, we have $C\left(N^{*}\right) .\left(N-N^{*}\right) \geq 0$. This latter form of the variational inequality will denote $\operatorname{VI}(C, K)$, where $C$ is the vector field and $K$ is the set of feasibles, both previously defined.

It should be noted that $C$ is not a conservative vector field given that $\partial c_{p} / \partial N_{p^{\prime}}=\sum_{a \in L}\left(\partial N_{a} / \partial N_{p^{\prime}}\right) c_{a}^{\prime}\left(N_{a}\right) \delta_{a p}$. This expression is not symmetric with respect to $p$ and $p^{\prime}$ in general, so the Wardrop conditions are not in general the optimality conditions of an optimization problem. This implies that we cannot write an equivalent optimization prob- lem (such as Beckmann's transformation) for the densitybased model.

Since the set $K$ is not in general closed due to the strict inequality $N_{a}<N_{a}^{\max }$, we define the set $K_{\varepsilon}$ for all $\varepsilon>0$ as

$$
\begin{aligned}
K_{\varepsilon} & =\left\{N=\left(N_{p}\right)_{p \in p} \mid N_{a}=\sum_{p \in P} N_{p} q_{a p}, N_{p} \geq 0, N_{a}\right. \\
& \left.\leq N_{a}^{\max }-\varepsilon, N^{w}=\sum_{p \in P^{w}} N_{p}\right\}
\end{aligned}
$$

Observe that $K_{\varepsilon} \subset K, K_{\varepsilon} \subset K_{\varepsilon^{\prime}}$ if $0<\varepsilon^{\prime}<\varepsilon$, and also $\bigcup_{\varepsilon>0} K_{\varepsilon}=K$. This implies that when $\varepsilon>0$ is small, $K_{\varepsilon}$ is a good approximation of the set $K$. Assume that the vector field $C$ is continuous in set $K$. Then it is also continuous in set $K_{\varepsilon}$ for $\varepsilon>0$, given that $K_{\varepsilon} \subset K$.

The following theorem gives an existence result for the inequality $\operatorname{VI}\left(C, K_{\varepsilon}\right)$, proving that there exists an $N^{*} \in K_{\varepsilon}$ such that, for all $N \in K_{\varepsilon}$, we have $C\left(N^{*}\right) .\left(N-N^{*}\right) \geq 0$.

Theorem 1 (let $\varepsilon>0$ ). If $K_{\varepsilon} \neq \varnothing$, then the variational inequality $\operatorname{VI}\left(C, K_{\varepsilon}\right)$ has at least one solution.

Proof. Recall that vector field $C$ is continuous in set $K_{\varepsilon}$. Observe further that $K_{\varepsilon}$ is closed and convex. We now prove that it is bounded.

Let $p$ be a route and $a$ a link such that $\delta_{a p}=1$. Given an $N \in K_{\varepsilon}$, the following inequality is true:

$$
N_{a}^{\max }-\varepsilon \geq N_{a}=\sum_{p^{\prime} \in P} N_{p^{\prime}} q_{a p^{\prime}} \geq N_{p} q_{a p}
$$

This implies that, for all $a$ on $p, N_{p} \leq\left(1 / q_{a p}\right)\left(N_{a}^{\max }-\right.$ $\varepsilon)$. Then $N_{p} \leq \min \left\{\left(1 / q_{a p}\right)\left(N_{a}^{\max }-\varepsilon\right) \mid \delta_{a p}=\right.$ 1\}. Furthermore, we know that $N_{p} \geq 0$. Finally, we have

$$
0 \leq N_{p} \leq \min \left\{\frac{1}{q_{a p}}\left(N_{a}^{\max }-\varepsilon\right) \mid \delta_{a p}=1\right\}
$$

This expression is satisfied for all $p$, which proves that $K_{\varepsilon}$ is bounded. Since it is also closed, we deduce that it is compact (i.e., closed and bounded). And, given that it is convex and (by hypothesis) nonempty as well, we conclude by Harker and Pang [43] that the variational inequality $\operatorname{VI}\left(C, K_{\varepsilon}\right)$ has at least one solution.

However, being a solution of $\operatorname{VI}\left(C, K_{\varepsilon}\right)$ is not a sufficient condition for satisfying Wardrop's first principle given that one of the $N_{a} \leq N_{a}^{\max }-\varepsilon$ restrictions may be active, which would imply the existence of a Lagrange multiplier that does not appear in the system of equations (5) expressing the principle. We must therefore also prove the existence of a solution of $\operatorname{VI}(C, K)$. This is done in Theorem 2 below. 
Before setting out the formal proof, however, we define $\bar{K}$ as the closure of set $K$ :

$$
\begin{aligned}
\bar{K} & =\left\{N=\left(N_{p}\right)_{p \in P} \mid N_{a}=\sum_{p \in P} N_{p} q_{a p}, N_{p} \geq 0, N_{a}\right. \\
& \left.\leq N_{a}^{\max }, N^{w}=\sum_{p \in P^{w}} N_{p}\right\}
\end{aligned}
$$

Theorem 2 (assume that $K \neq \varnothing$ ). If $K=\bar{K}$, or if, for all $N^{*} \in$ $\bar{K} \backslash K$, for every sequence $N^{n} \in K$ that satisfies $\lim _{n \rightarrow \infty} N^{n}=$ $N^{*}$, there exists an element $N \in K$ such that

$$
\lim _{n \rightarrow \infty} C\left(N^{n}\right) .\left(N-N^{n}\right)<0
$$

then $\operatorname{VI}(C, K)$ has at least one solution.

Proof. Given that $K$ is a bounded and convex set, the set $\bar{K}$ is compact and convex.

If $K=\bar{K}$, then $K$ is a compact, convex set. Given that $C$ is continuous in $K$, we deduce that $\operatorname{VI}(C, K)$ has at least one solution.

Assume that $\neq \bar{K}$; that is, $\bar{K} \backslash K \neq \varnothing$. Given that $K \neq \varnothing$, then $K_{1 / n} \neq \varnothing$ for any sufficiently large $n$, and, therefore, by Theorem $1, \operatorname{VI}\left(C, K_{1 / n}\right)$ has at least one solution.

Let $N^{n}$ be a solution of $\operatorname{VI}\left(C, K_{1 / n}\right)$. Given that the set $\bar{K}$ is compact, the sequence $N^{n}$ has a subsequence $N^{n_{k}}$ that converges. We denote by $N^{*} \in \bar{K}$ the limit of the sequence $N^{n_{k}}$. Consider the following two cases:

Case $1\left(N^{*} \in K\right)$. Let $N \in K$. Given that $K=\bigcup_{n \geq 1} K_{1 / n}$, there exists a $k_{0} \geq 1$ such that, for all $k \geq k_{0}, N \in K_{1 / n_{k}}$. Given that $N^{n_{k}}$ is a solution of $\operatorname{VI}\left(C, K_{1 / n_{k}}\right)$, we have $C\left(N^{n_{k}}\right) .(N-$ $\left.N^{n_{k}}\right) \geq 0$. Since $C$ is a continuous vector field in $K$, we have

$$
C\left(N^{*}\right) \cdot\left(N-N^{*}\right)=\lim _{k \rightarrow \infty} C\left(N^{n_{k}}\right) \cdot\left(N-N^{n_{k}}\right) \geq 0
$$

Then $N^{*}$ is a solution of $\operatorname{VI}(C, K)$.

Case $2\left(N^{*} \in \bar{K} \backslash K\right)$. Let $N \in K$ such that $\lim _{k \rightarrow \infty} C\left(N^{n_{k}}\right) .\left(N-N^{n_{k}}\right)<0$ ( $N$ exists by hypothesis since $\lim _{k \rightarrow \infty} N^{n_{k}}=N^{*}$ and $\left.N^{*} \in \bar{K} \backslash K\right)$. Given that $\in K_{1 / n_{k}}$, for all sufficiently large $k$ and that $N^{n_{k}}$ is a solution of $\operatorname{VI}\left(C, K_{1 / n_{k}}\right)$, then $C\left(N^{n_{k}}\right) \cdot\left(N-N^{n_{k}}\right) \geq 0$ for any sufficiently large $k$, which contradicts the inequality $\lim _{k \longrightarrow \infty} C\left(N^{n_{k}}\right) .\left(N-N^{n_{k}}\right)<0$. Therefore, Case 2 cannot occur.

Thus, only Case 1 can occur, which implies the existence of at least one solution of $V I(C, K)$.

A proposition for a general example of a network that satisfies condition (11) is given in Appendix B.

About the uniqueness, the following theorem gives us that the quantity of vehicles of arc is unique and, then, the cost of the route is also unique.
Theorem 3. Let $N^{*}$ and $M^{*}$ be two solutions of $V I(C, K)$. For each link $a$, we have $N_{a}^{*}=M_{a}^{*}$, and for each route $p$, we have $c_{p}\left(N^{*}\right)=c_{p}\left(M^{*}\right)$.

Proof (Let $N \in K)$. We have $C\left(N^{*}\right) .\left(N-N^{*}\right) \geq 0$ which implies that $\sum_{p} c_{p}\left(N^{*}\right) \cdot\left(N_{p}-N_{p}^{*}\right) \geq 0$. Given that $c_{p}\left(N_{p}\right)=$ $\sum_{a} c_{a}\left(N_{a}\right) \delta_{a p}$, we have

$$
\begin{aligned}
\sum_{p} c_{p}\left(N^{*}\right) \cdot\left(N_{p}-N_{p}^{*}\right) \\
=\sum_{p}\left(\sum_{a} c_{a}\left(N_{a}^{*}\right) \delta_{a p}\right) \cdot\left(N_{p}-N_{p}^{*}\right) \\
=\sum_{a} c_{a}\left(N_{a}^{*}\right)\left(\sum_{p} \delta_{a p}\left(N_{p}-N_{p}^{*}\right)\right) \\
=\sum_{a} c_{a}\left(N_{a}^{*}\right) \cdot\left(N_{a}-N_{a}^{*}\right)
\end{aligned}
$$

This implies that

$$
\begin{aligned}
\forall N & \in K^{\prime}, \\
\sum_{a} c_{a}\left(N_{a}^{*}\right) \cdot\left(N_{a}-N_{a}^{*}\right) & \geq 0
\end{aligned}
$$

Where

$$
\begin{aligned}
& K^{\prime} \\
& =\left\{N^{\prime}=\left(N_{a}\right) \mid \exists N \in K, \forall a \in L, N_{a}=\sum_{p} N_{p} q_{a p}\right\}
\end{aligned}
$$

Define $C^{\prime}\left(N^{\prime}\right)=\left(c_{a}\left(N_{a}\right)\right)_{a \in L}$. From what we stand before, the vector $\left(N_{a}^{*}\right)$ is a solution of $\operatorname{VI}\left(C^{\prime}, K^{\prime}\right)$. By the same way, the vector $\left(M_{a}^{*}\right)$ is a solution of $V I\left(C^{\prime}, K^{\prime}\right)$. Given that each function $c_{a}$ is strictly increasing, we deduce that the vector field $C^{\prime}$ is strictly monotone. Moreover, it is clear that $K^{\prime}$ is a convex set; then $V I\left(C^{\prime}, K^{\prime}\right)$ admits a unique solution. We deduce that, for each $\operatorname{arc} a$, we have $N_{a}^{*}=M_{a}^{*}$.

We deduce that, for each $\operatorname{arc} a$, we have $c_{a}\left(N_{a}^{*}\right)=c_{a}\left(M_{a}^{*}\right)$, and then $c_{p}\left(N^{*}\right)=c_{p}\left(M^{*}\right)$.

A big issue for this model is the fact that if we consider $q_{a p}=\left(t_{a} / t_{p}\right) \delta_{a p}$ (this choice allows for flow conservation to be satisfied), then $q_{a p}$ depend on $N_{a}$ which depends on $q_{a p}$. In what follows, we propose an algorithm for constructing iteratively a solution of $\operatorname{VI}(C, K)$ with $q_{a p}=\left(t_{a} / t_{p}\right) \delta_{a p}$. In the sequel, we consider $\alpha_{a}>0$ and $\beta_{a}>0$ such that the average speed on link $a$ is given by $v_{a}=\alpha_{a}-\beta_{a} D_{a}$, with $D_{a}=N_{a} / l_{a}$ the density of vehicles present on link a. In this case we have $t_{a}=l_{a} /\left(\alpha_{a}-\beta_{a} D_{a}\right)$.

We consider the function $t_{a}\left(N_{a}\right)=l_{a} /\left(\alpha_{a}-\beta_{a} D_{a}\right)=$ $l_{a}^{2} /\left(l_{a} \alpha_{a}-\beta_{a} N_{a}\right)$, which is the average time for travelling the link $a$ if there are $N_{a}$ vehicles, and the function $t_{p}(N)=\sum_{a \in L} t_{a}\left(N_{a}\right) \delta_{a p}$ which is the average time for travelling the route $p$ if the vector of quantities of vehicles is $N=\left(N_{p}\right)_{p}$. 
Algorithm 4. We construct the sequence $\left(N^{n}\right)$ by the following way.

Step 0. Let $t_{a}^{0}=l_{a} / \alpha_{a}, t_{p}^{0}=\sum_{a} t_{a}^{0} \delta_{a p}, q_{a p}^{0}=\left(t_{a}^{0} / t_{p}^{0}\right) \delta_{a p}, n=1$. Let $\varepsilon>0$.

Step 1 . Given the quantity $q_{a p}^{n-1}$, consider the set

$$
\begin{aligned}
K^{n} & =\left\{N=\left(N_{p}\right)_{p \in P} \mid N_{a}=\sum_{p \in P} N_{p} q_{a p}^{n-1}, N_{p} \geq 0, N_{a}\right. \\
& \left.<N_{a}^{\max }, N^{w}=\sum_{p \in P^{w}} N_{p}\right\} .
\end{aligned}
$$

Construct $N^{n}=\left(N_{p}^{n}\right)_{p \in P}$ as a solution of $\operatorname{VI}\left(C, K^{n}\right)$.

Step 2. Consider $N_{a}^{n}=\sum_{p \in P} N_{p}^{n} q_{a p}^{n-1}$ the quantity of vehicles on link $a, f_{a}=N_{a}^{n} / t_{a}\left(N_{a}^{n}\right)$ the vehicles flow on link $a$, and $h_{p}=N_{p}^{n} / \sum_{a} t_{a}\left(N_{a}^{n}\right) \delta_{a p}$ the vehicles flow on route $p$. If $\sum_{a \in L}\left(f_{a}-\sum_{p \in P} h_{p} \delta_{a p}\right)^{2} / \sum_{a \in L}\left(f_{a}\right)^{2}<\varepsilon^{2}$, then stop; else, go to step 3.

Step 3. Construct $\left(N_{a}\right)_{a}$ as a solution of the equations $N_{a}=$ $\sum_{p \in P}\left(t_{a}\left(N_{a}\right) / \sum_{a^{\prime} \in L} t_{a^{\prime}}\left(N_{a^{\prime}}\right) \delta_{a^{\prime} p}\right) N_{p}^{n} \delta_{a p}$. Consider $q_{a p}^{n}=$ $\left(t_{a}\left(N_{a}\right) / \sum_{a^{\prime} \in L} t_{a^{\prime}}\left(N_{a^{\prime}}\right) \delta_{a^{\prime} p}\right) \delta_{a p}, n=n+1$, and go to Step 1.

Step 2 tests whether the relative error in the flow conservation law is small enough. This algorithm has been implemented to obtain the results of the following section.

\subsection{Estimation of Road Project Benefits and Summary of Model} Characteristics. The proposed model can be used to conduct a social cost-benefit analysis of a road system project. If we let $C_{0}^{w}$ be the equilibrium cost before the project is implemented and let $C_{1}^{w}$ be the equilibrium cost after implementation, the change in consumer surplus $(\triangle E C)$ is estimated directly by the following formula:

$$
\begin{aligned}
\Delta E C & =\sum_{w} \int_{C_{0}^{w}}^{C_{1}^{w}} T^{w} d C^{w}=\sum_{w} \int_{C_{0}^{w}}^{C_{1}^{w}} \frac{N^{w}}{C^{w}} d C^{w} \\
& =\sum_{w} N^{w} \ln \left(\frac{C_{1}^{w}}{C_{0}^{w}}\right)
\end{aligned}
$$

where $T^{w}$ is the trip matrix (vehicle flows per hour). In the classical flow-based model, on the other hand, where $T^{w}$ is exogenous, the change in consumer surplus is given by

$$
\triangle E C=T^{w}\left(C_{1}^{w}-C_{0}^{w}\right)
$$

Briefly, in (17) the social benefit of a transport project or policy derives from the greater number of individuals benefiting in the same period of time whereas in (18) the social benefit consists in the same number of individuals experiencing lower trip costs.

A summary of the main characteristics of the classical flow-based and proposed density-based models and the differences between them is laid out in Table 1 .

\section{Comparative Application of Models and Results}

In this section we discuss the application of the classical flowbased and proposed density-based models to a numerical example of a road network and compare their results. Both low and high congestion scenarios are considered. The two approaches are also used to estimate the benefits of a road system project. Notice that, in the absence of congestion, the density-based model will always provide identical results as the flow-based model.

4.1. The Nguyen-Dupuis Network. The road network assumed for this application is an adaptation of the one developed by Nguyen and Dupuis [44], depicted here in Figure 4. It consists of 13 nodes and 19 links. Four O-D pairs are defined: A-K, A$\mathrm{M}, \mathrm{C}-\mathrm{K}$, and $\mathrm{C}-\mathrm{M}$. There are 23 different possible routes for the four pairs as set out in Table 2 .

We define the relationship between speed and density on each link defined by (1) as $V_{a}=50-D_{a}$. This implies that the free-flow speed is $50 \mathrm{~km} / \mathrm{h}$, the density that generates the maximum flow is 25 vehicles per kilometre $\left(D_{a}^{*}=\right.$ 25 ), and the maximum density is 50 vehicles per kilometre $\left(D_{a}^{\max }=50\right)$. The lengths in kilometres of the 19 links, ordered by number as shown in Figure 4, are as follows: $l_{a}=$ $\left[\begin{array}{lllllllllllllllllll}5 & 1 & 10 & 3 & 2 & 9 & 2 & 10 & 5 & 10 & 2 & 2 & 2 & 2 & 2 & 3 & 3 & 2 & 2\end{array}\right]$. The maximum flow is $f_{a}^{\max }=625$.

For the proposed density-based model, the flow of vehicles travelling between each of the four O-D pairs is defined by matrices $T_{1}$ and $T_{2}$ representing, respectively, the high congestion case and the low congestion case, as follows:

$$
\begin{aligned}
T_{1} & =\left[\begin{array}{llll}
T_{A-K} & T_{A-M} & T_{C-K} & T_{C-M}
\end{array}\right] \\
& =\left[\begin{array}{llll}
810 & 400 & 450 & 800
\end{array}\right] \\
T_{2} & =\left[\begin{array}{llll}
T_{A-K} & T_{A-M} & T_{C-K} & T_{C-M}
\end{array}\right] \\
& =\left[\begin{array}{llll}
500 & 500 & 500 & 500
\end{array}\right]
\end{aligned}
$$

In order to compute the quantity of vehicles $N^{w}$, which are travelling the O-D pair $w$, we solve the flow-based model. To achieve this goal, we solve the equation

$$
H(h, \lambda, \mu)=\left(\begin{array}{c}
C(h)+\Delta^{t} \lambda-\mu \\
\Delta h-T \\
h \otimes \mu
\end{array}\right)=0
$$

with $h \geq 0$ and $\mu \geq 0$. Notice that $\Delta$ is the OD-route incidence matrix, $h=\left(h_{p}\right)$ is the vector of flow on routes, $T=\left(T_{w}\right)$ is the vector of flow demands, $\otimes$ is the Hadamard product which is defined as $h \otimes \mu=\left(h_{p} \cdot \mu_{p}\right)$, and $C(h)=\left(C_{p}(h)\right)$ is the vector of cost on routes using flow-based model.

The gap function that we consider is $\|H(h, \lambda, \mu)\|$. The convergence criterion we use is $\|H(h, \lambda, \mu)\|<10^{-4}$. The gap function is necessary to guarantee the convergence of the results of the models in the equilibrium. Our gap function, specifically, measures how much Wardrop's balance is violated. 
Table 1: Summary of Main Differences between Flow-Based and Density-Based Models.

\begin{tabular}{|c|c|c|}
\hline Characteristic & Flow-Based Model & Density-Based Model \\
\hline General concept & $\begin{array}{l}\text { Assigns vehicles per hour among the } \\
\text { different network links for a } \\
\text { representative time period. }\end{array}$ & $\begin{array}{l}\text { Assigns vehicles per kilometre among } \\
\text { the different network links for a } \\
\text { representative instant in time. }\end{array}$ \\
\hline Equilibrium condition & $\begin{array}{l}\text { Satisfies Wardrop's first principle for all } \\
\text { network routes. }\end{array}$ & $\begin{array}{l}\text { Satisfies Wardrop's first principle for all } \\
\text { network routes. }\end{array}$ \\
\hline State of system & $\begin{array}{c}\text { Assumes that all trips in the trip matrix } \\
\text { are assigned to the network and that all } \\
\text { trips enter and exit within a given } \\
\text { modelled period. }\end{array}$ & $\begin{array}{l}\text { Assumes that the total number of } \\
\text { vehicles on the network at a given instant } \\
\text { is the same at the instant immediately } \\
\text { preceding and immediately following. }\end{array}$ \\
\hline Link capacity & $\begin{array}{l}\text { Each link is assumed to have a fixed, } \\
\text { exogenous capacity or maximum flow. } \\
\text { This capacity will often be exceeded. }\end{array}$ & $\begin{array}{l}\text { Each link's maximum flow, rather than } \\
\text { being a fixed parameter, depends on } \\
\text { vehicle density and speed across it and } \\
\text { will vary with traffic conditions. } \\
\text { Maximum flow is finite. }\end{array}$ \\
\hline Trip matrix & $\begin{array}{l}\text { Assumes a trip matrix (flows between } \\
\text { O-D pairs) for the period modelled that is } \\
\text { fixed and exogenous to equilibrium cost. }\end{array}$ & $\begin{array}{l}\text { Assumes the number of vehicles for the } \\
\text { instant modelled is fixed and exogenous } \\
\text { but the trip matrix (flows between O-D } \\
\text { pairs) is a result of the equilibrium. }\end{array}$ \\
\hline Conservation of flow & $\begin{array}{c}\text { By construction, assumes conservation of } \\
\text { flow at all network nodes including cases } \\
\text { where route time or cost is greater than } \\
\text { the period modelled (i.e., there are } \\
\text { travellers who do not arrive at their } \\
\text { destination). }\end{array}$ & $\begin{aligned} \text { With } q_{a p}=\left(t_{a} / t_{p}\right) \delta_{a p} \text { the conservation } \\
\text { of flow is satisfied. }\end{aligned}$ \\
\hline Densities and queue lengths under congestion & $\begin{array}{l}\text { Does not satisfactorily estimate either } \\
\text { densities or queue lengths under high } \\
\text { congestion. }\end{array}$ & $\begin{array}{c}\text { Always correctly estimates densities and } \\
\text { queue lengths, whether under low or } \\
\text { high congestion. }\end{array}$ \\
\hline Fundamental traffic equation & $\begin{array}{l}\text { Under high congestion, the } f=V \cdot D \\
\text { relationship does not hold. }\end{array}$ & $\begin{array}{l}\text { By construction, the } f=V \cdot D \\
\text { relationship always holds. }\end{array}$ \\
\hline Estimation of benefits of road system project or policy & $\begin{array}{c}\text { Benefits are estimated in terms of } \\
\text { network user time savings. }\end{array}$ & $\begin{array}{l}\text { Benefits are estimated as the change in } \\
\text { consumer surplus. }\end{array}$ \\
\hline
\end{tabular}

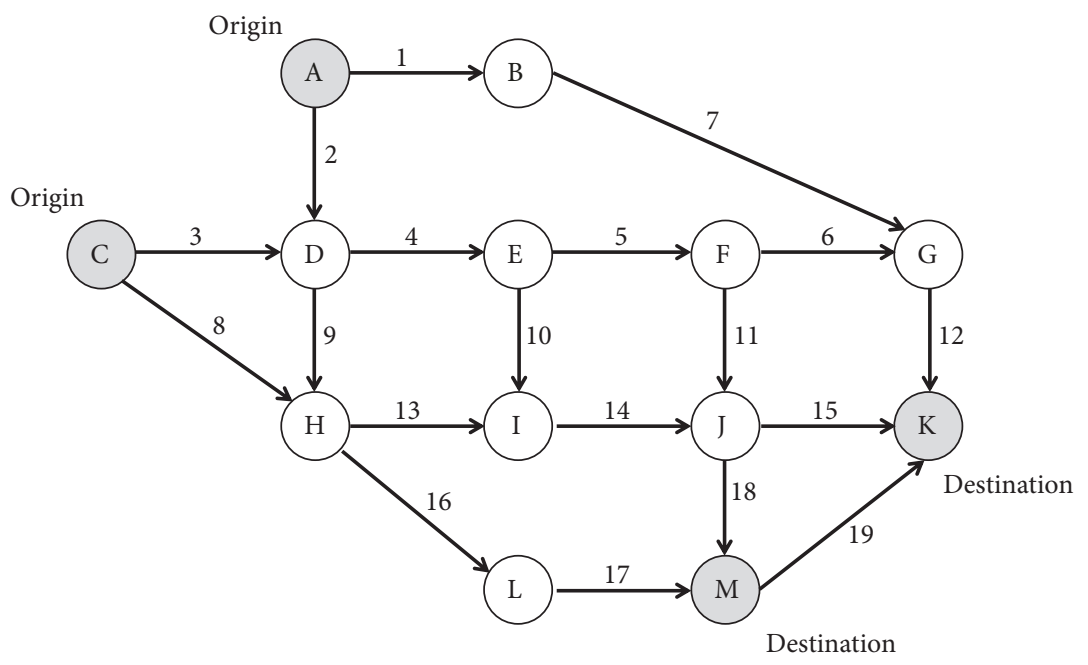

FIgURE 4: Nguyen-Dupuis Network (Adaptation). 


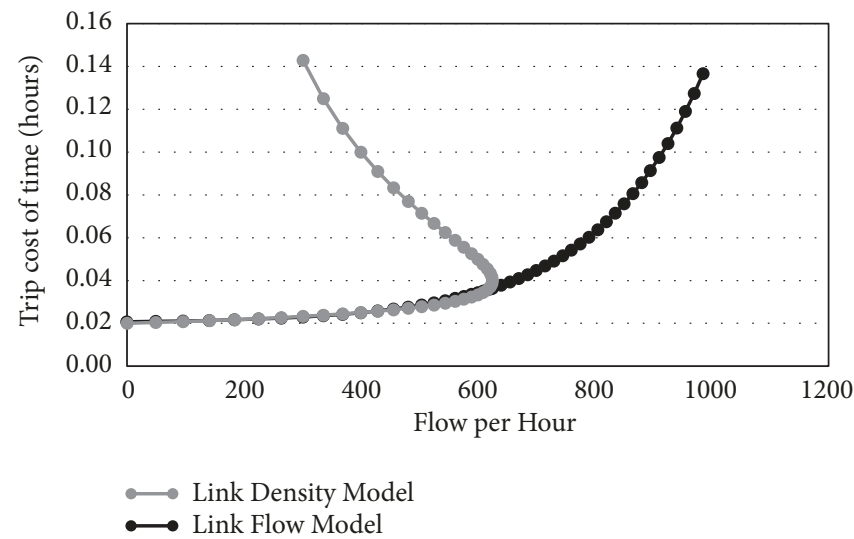

FIgUre 5: Flow-Delay Curves for Flow-Based and Density-Based Approaches.

Table 2: Possible Routes for Origin-Destination Pairs.

\begin{tabular}{lcc}
\hline Route No. & O-D Pair & Route (link sequence) \\
\hline 1 & A-K & $1-7-12$ \\
2 & A-K & $2-4-5-6-12$ \\
3 & A-K & $2-4-5-11-15$ \\
4 & A-K & $2-4-10-14-15$ \\
5 & A-K & $2-9-13-14-15$ \\
6 & A-K & $2-9-13-14-18-19$ \\
7 & A-K & $2-9-16-17-19$ \\
8 & A-M & $2-4-5-11-18$ \\
9 & A-M & $2-4-10-14-18$ \\
10 & A-M & $2-9-13-14-18$ \\
11 & A-M & $2-9-16-17$ \\
12 & C-K & $3-4-5-6-12$ \\
13 & C-K & $3-4-5-11-15$ \\
14 & C-K & $3-4-10-14-15$ \\
15 & C-K & $3-9-13-14-15$ \\
16 & C-K & $8-13-14-15$ \\
17 & C-K & $8-13-14-18-19$ \\
18 & C-K & $8-16-17-19$ \\
19 & C-M & $3-4-5-11-18$ \\
20 & C-M & $3-4-10-14-18$ \\
21 & C-M & $3-9-13-14-18$ \\
22 & C-M & $8-16-17$ \\
23 & C-M &
\end{tabular}

We solved (20) using a reduction potential method [45]. For this approach we estimate flow-delay functions that must satisfy two conditions. First, they must be as similar as possible to the flow-delay functions used for the density-based assignments and, second, they have to be monotonically increasing.

A function that meets both conditions is the black curve shown in Figure 5, where it is graphed together with the grey curve representing the flow-delay relationship of our density-based model. The grey curve is constructed assuming parameter values $\alpha_{a}=50, \beta_{a}=1$, for a link of length $l_{a}=1$. For the black curve, on the other hand, each link $a$ is defined by an exponential function of the form $c_{a}\left(f_{a}\right)=t_{a}^{0}+l_{a} \eta_{a} \exp \left(\rho_{a} \cdot f_{a}\right)$, where $t_{a}^{0}$ is the free-flow time of $a$ and $\left[\eta_{a}, \rho_{a}\right]$ are positive parameters estimated so as to follow as closely as possible the increasing segment of the grey curve. The actual parameter values used are $\eta_{a}=$ $0.00053302, \rho_{a}=0.0054674137$. With these two flow-delay functions representing the two approaches, we would expect them to produce similar results in low congestion situations but significantly different results with high congestion.

Using the same $T_{1}$ (high congestion) and $T_{2}$ (low congestion) matrix values given in (19) and the corresponding equilibrium costs for each O-D pair (Tables 3 and 5), we estimate two vectors $N_{1}$ and $N_{2}$ of quantity of vehicles travelling between each of the four $\mathrm{O}-\mathrm{D}$ pairs multiplying the vectors of flows $T_{1}$ and $T_{2}$ by the time for travelling the $\mathrm{O}$ $\mathrm{D}$ pairs computed using the flow-based model. We obtain, for high congestion, the following time for travelling the O-D pairs with flow-based model:

$$
\begin{aligned}
c_{1} & =\left[\begin{array}{llll}
c_{A-K} & c_{A-M} & c_{C-K} & c_{C-M}
\end{array}\right] \\
& =\left[\begin{array}{llll}
0.3603 & 0.3629 & 0.6387 & 0.6413
\end{array}\right] \\
c_{2} & =\left[\begin{array}{llll}
c_{A-K} & c_{A-M} & c_{C-K} & c_{C-M}
\end{array}\right] \\
& =\left[\begin{array}{llll}
0.2538 & 0.3075 & 0.5201 & 0.5219
\end{array}\right]
\end{aligned}
$$

The quantity of vehicles travelling between each of the four O-D pairs is defined by matrices $N_{1}$ and $N_{2}$ representing, respectively, the high congestion case and the low congestion case, as follows:

$$
\begin{aligned}
& N_{1} \\
& =\left[\begin{array}{llll}
810 \times 0.3603 & 400 \times 0.3629 & 450 \times 0.6387 & 800 \times 0.6413
\end{array}\right] \\
& =\left[\begin{array}{llll}
291.82 & 145.16 & 287.41 & 513.05
\end{array}\right] \\
& N_{2} \\
& =\left[\begin{array}{llll}
500 \times 0.2538 & 500 \times 0.3075 & 500 \cdot \times 0.5201 & 500 \times 0.5219
\end{array}\right] \\
& =\left[\begin{array}{llll}
126.91 & 153.75 & 260.04 & 260.97
\end{array}\right]
\end{aligned}
$$


TABle 3: Equilibrium Route Assignments, Density-Based Approach (high congestion).

\begin{tabular}{lccc}
\hline Route & O-D Pair & $N_{p}$ & $C_{p}$ \\
\hline 1 & A-K & 224.3485 & 0.3592 \\
2 & A-K & 0.0000 & 0.4872 \\
3 & A-K & 36.4514 & 0.3592 \\
4 & A-K & 0.0000 & 0.4919 \\
5 & A-K & 31.0230 & 0.3592 \\
6 & A-K & 0.0000 & 0.3937 \\
7 & A-K & 0.0000 & 0.3937 \\
8 & A-M & 41.6268 & 0.3537 \\
9 & A-M & 0.0000 & 0.4864 \\
10 & A-M & 34.6943 & 0.3537 \\
11 & A-M & 68.8369 & 0.3537 \\
12 & C-K & 0.0000 & 0.8222 \\
13 & C-K & 144.2187 & 0.6942 \\
14 & C-K & 0.0000 & 0.8269 \\
15 & C-K & 85.1820 & 0.6942 \\
16 & C-K & 58.0120 & 0.6942 \\
17 & C-K & 0.0000 & 0.7288 \\
18 & C-K & 0.0000 & 0.7288 \\
19 & C-M & 126.0871 & 0.6888 \\
20 & C-M & 0.0000 & 0.8214 \\
21 & C-M & 78.5134 & 0.6888 \\
22 & C-M & 54.8398 & 0.6888 \\
23 & C-M & 253.6105 & 0.6888 \\
\hline & & &
\end{tabular}

We solve the density-based model solving the equation

$$
H(N, \lambda, \mu)=\left(\begin{array}{c}
C(N)+\Delta^{t} \lambda-\mu \\
\Delta N-\left(N_{w}\right) \\
N \otimes \mu
\end{array}\right)=0
$$

with $N \geq 0$ and $\mu \geq 0$. Notice that $\Delta$ is the OD-route incidence matrix, $N=\left(N_{p}\right)$ is the vector of quantities on routes, $\left(N_{w}\right)$ is the vector of quantities demands between OD pairs, $N \otimes \mu=\left(N_{p} \cdot \mu_{p}\right)$, and $C(N)=\left(C_{p}(N)\right)$ is the vector of cost on routes using density-based model. The gap function we use is $\|H(N, \lambda, \mu)\|$. The convergence criterion we use is $\|H(N, \lambda, \mu)\|<10^{-4}$.

For high congestion scenario, the gap function for density-based model is equal at the equilibrium to 0.7614 . $10^{-10}$. The equilibrium route and link assignment results for the high congestion case are set out in Tables 3 and 4. From Table 3 we see that, for O-D pair A-K, 3 of the 7 possible routes are used and all 3 have the same cost, satisfying Wardrop's first principle (routes 2, 4, 6, and 7 are not used, show higher costs). Regarding the other three pairs, 3 of the 4 possible routes are used for A-M, 3 of the possible 7 are used for C$\mathrm{K}$, and 4 of the possible 5 are used for C-M.

Table 4 shows that 3 of the 19 links are not used and thus their vehicle numbers and flows are 0 . Also apparent is that 4 links $(1,4,7$, and 8$)$ have densities greater than the critical density level $D_{a}^{*}=25$. This means that these links' flows are located on the decreasing segment of the curve in Figure 1.

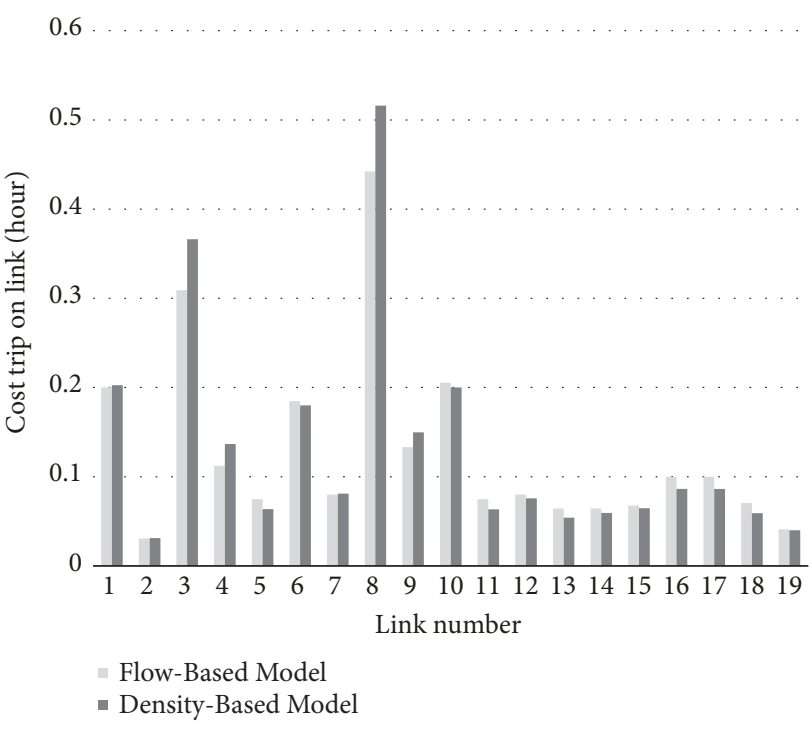

Figure 6: Link Costs, Both Approaches (high congestion).

For the low congestion case, Table 5 shows that 1 of the 7 possible routes is used for pair $\mathrm{A}-\mathrm{K}, 3$ of the 4 possible routes are used for A-M, 3 of the 7 possible routes are used for $\mathrm{C}-\mathrm{K}$, and 4 of the 5 possible routes are used for C-M. According to Table 6 , of the 19 links there are 3 unused and no link has a density greater than the maximum flow level $D_{a}^{*}$. Thus, the flows on these links are located on the increasing segment of the curve in Figure 1. In this case, the gap function for densitybased model is equal at the equilibrium to $0.7756 \cdot 10^{-10}$.

4.2. Comparison of Density-Based Model with Flow-Based Model. We now compare the above results for the densitybased model with those of a classical flow-based model.

We would expect the link flow and cost results obtained for $T_{1}$ under this approach to differ significantly from those obtained above for $N_{1}$ under the density-based approach, reflecting the difference between the two approaches when congestion is high. When $T_{2}$ and $N_{2}$ are compared, however, the link flow and cost results should be relatively similar, mirroring the greater similarity between the two approaches when congestion is low.

The link costs and flows under the two approaches for the high congestion case are brought together in Table 7 and the two sets of values are charted separately in Figures 6 and 7. As can be seen in the table, both flows and costs for the two models are significantly different. Some of the links under the flow-based model have flows considerably greater than the theoretical capacity of 625 (links 1, 4, 5, 7, 8, 11, and 12), whereas, under the density-based model, this never occurs. Costs under the two approaches also differ appreciably, the density-based model always showing higher cost levels.

A metric which can compare both models is the relative distance between the link costs and the link flows of the density-based model and the flow-based model. More precisely, we can consider the following quantities, where $f^{D}$ and $f^{F}$ are the flow vectors in, respectively, the density-based 
TABle 4: Equilibrium Link Assignments, Density-Based Approach (high congestion).

\begin{tabular}{lccccccc}
\hline Link & $N_{a}$ & $l_{a}$ & $D_{a}$ & $D_{a}^{*}$ & $V_{a}$ & $c_{a}$ & $f_{a}$ \\
\hline 1 & 126.5223 & 5 & 25.3045 & 25 & 24.6955 & 0.2025 & 624.9073 \\
2 & 17.9292 & 1 & 17.9292 & 25 & 32.0708 & 0.0312 & 575.0042 \\
3 & 226.9215 & 10 & 22.6921 & 25 & 27.3079 & 0.3662 & 619.6738 \\
4 & 84.1265 & 3 & 28.0422 & 25 & 21.9578 & 0.1366 & 615.7452 \\
5 & 37.1041 & 2 & 18.5520 & 25 & 31.4480 & 0.0636 & 583.4237 \\
6 & 0.0000 & 9 & 0.0000 & 25 & 50.0000 & 0.1800 & 0.0000 \\
7 & 50.6089 & 2 & 25.3045 & 25 & 24.6955 & 0.0810 & 624.9073 \\
8 & 306.1953 & 10 & 30.6195 & 25 & 19.3805 & 0.5160 & 593.4209 \\
9 & 83.0978 & 5 & 16.6196 & 25 & 33.3804 & 0.1498 & 554.7684 \\
10 & 0.0000 & 10 & 0.0000 & 25 & 50.0000 & 0.2000 & 0.0000 \\
11 & 36.7618 & 2 & 18.3809 & 25 & 31.6191 & 0.0633 & 581.1875 \\
12 & 47.2174 & 2 & 23.6087 & 25 & 26.3913 & 0.0758 & 623.0643 \\
13 & 26.1439 & 2 & 13.0720 & 25 & 36.9280 & 0.0542 & 482.7217 \\
14 & 32.8045 & 2 & 16.4023 & 25 & 33.5977 & 0.0595 & 551.0790 \\
15 & 38.0592 & 2 & 19.0296 & 25 & 30.9704 & 0.0646 & 589.3545 \\
16 & 45.8212 & 3 & 15.2737 & 25 & 34.7263 & 0.0864 & 530.3997 \\
17 & 45.8212 & 3 & 15.2737 & 25 & 34.7263 & 0.0864 & 530.3997 \\
18 & 32.3097 & 2 & 16.1549 & 25 & 33.8451 & 0.0591 & 546.7634 \\
19 & 0.0000 & 2 & 0.0000 & 25 & 50.0000 & 0.0400 & 0.0000 \\
\hline
\end{tabular}

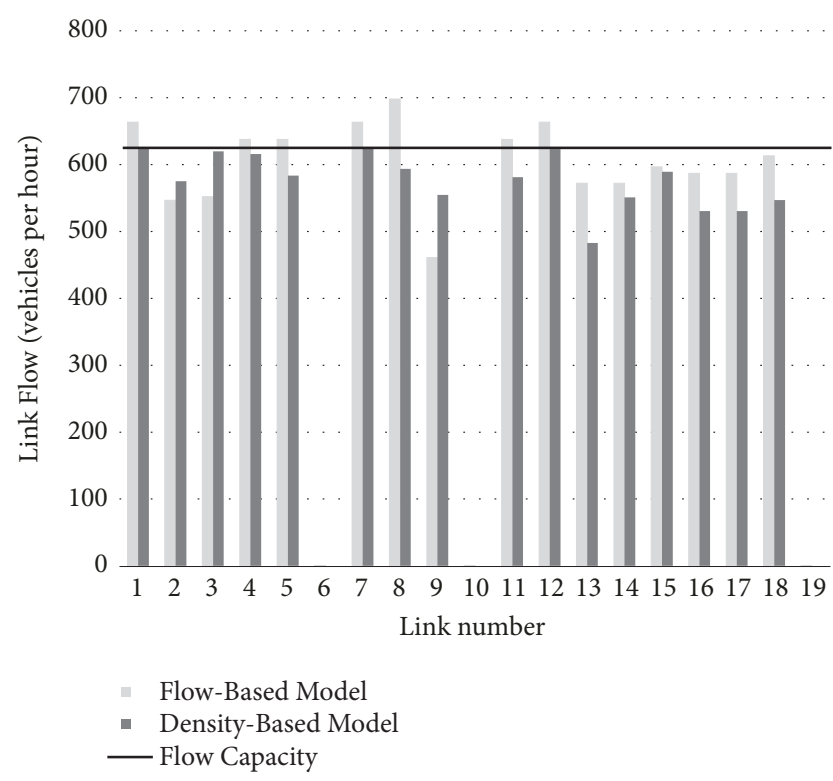

Figure 7: Link Flows, Both Approaches (high congestion).

model and the flow-based model, where $c^{D}$ and $c^{F}$ are the flow vectors in, respectively, the density-based model and the flow-based model:

$$
\begin{aligned}
\operatorname{dist}\left(f^{D}, f^{f}\right) & =2 \frac{\left\|f^{D}-f^{F}\right\|}{\left\|f^{D}+f^{F}\right\|}, \\
\operatorname{dist}\left(c^{D}, c^{f}\right) & =2 \frac{\left\|c^{D}-c^{F}\right\|}{\left\|c^{D}+c^{F}\right\|}
\end{aligned}
$$

We can observe that both quantities are relative distances between the results obtained with the density-based model and the flow-based model. The relative distances that we obtain for the high congestion case are

$$
\begin{array}{r}
\operatorname{dist}\left(f^{D}, f^{F}\right)=0.0994, \\
\operatorname{dist}\left(c^{D}, c^{F}\right)=0.1382
\end{array}
$$

For the low congestion case, the link costs and flows under the flow-based (matrix $T_{2}$ ) and density-based (matrix $N_{2}$ ) approaches are given in Table 8, and the two sets of values are charted separately in Figures 8 and 9. The table shows that both flows and costs for each model are relatively similar. Note, however, that on every link the cost under the densitybased model is equal to or higher than that for the flowbased model. The relative distances that we obtain for the low congestion case are

$$
\begin{aligned}
\operatorname{dist}\left(f^{D}, f^{f}\right) & =0.0303, \\
\operatorname{dist}\left(c^{D}, c^{f}\right) & =0.0219
\end{aligned}
$$

These data for the two cases confirm our expectation that the results of the two approaches are similar under low congestion but differ when congestion is high.

The relative difference obtained for costs and flows in both scenarios are reported in Table 9. They confirm that, as congestion declines, link costs and flows under the two approaches grow increasingly similar but, as congestion rises, they increasingly diverge.

Finally, the gap function for flow-based model with high congestion is $9.1915 \cdot 10^{-4}$, and for low congestion is 9.4065 . $10^{-4}$. 
TABle 5: Equilibrium Route Assignments, Density-Based Approach (low congestion).

\begin{tabular}{lccc}
\hline Route & O-D Pair & $N_{p}$ & $C_{p}$ \\
\hline 1 & A-K & 126.9098 & 0.2507 \\
2 & A-K & 0.0000 & 0.4214 \\
3 & A-K & 0.0000 & 0.3031 \\
4 & A-K & 0.0000 & 0.4280 \\
5 & A-K & 0.0000 & 0.3031 \\
6 & A-K & 0.0000 & 0.3451 \\
7 & A-K & 0.0000 & 0.3451 \\
8 & A-M & 77.0756 & 0.3051 \\
9 & A-M & 0.0000 & 0.4300 \\
10 & A-M & 24.9098 & 0.3051 \\
11 & A-M & 51.7650 & 0.3051 \\
12 & C-K & 0.0000 & 0.6404 \\
13 & C-K & 122.2635 & 0.5221 \\
14 & C-K & 0.0000 & 0.6470 \\
15 & C-K & 28.2865 & 0.5221 \\
16 & C-K & 109.4890 & 0.5221 \\
17 & C-K & 0.0000 & 0.5640 \\
18 & C-K & 0.0000 & 0.5640 \\
19 & C-M & 38.6385 & 0.5240 \\
20 & C-M & 0.0000 & 0.6489 \\
21 & C-M & 18.8462 & 0.5240 \\
22 & C-M & 37.2549 & 0.5240 \\
23 & C-M & 166.2343 & 0.5240 \\
\hline
\end{tabular}

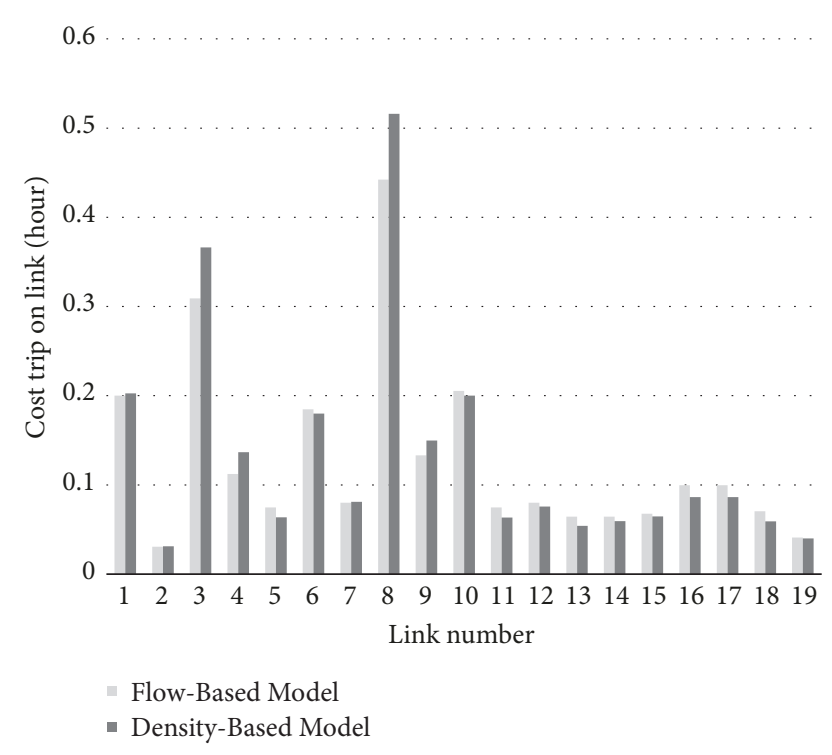

FIgURE 8: Link Costs, Both Approaches (low congestion).

4.3. Obtaining the $N^{w}$ Matrix Elements. Both the flow matrix containing the $T^{w}$ and the vehicle matrix containing the $N^{w}$ can be obtained from trip surveys that capture trip origins and destinations and the corresponding departure and arrival times. To estimate the $N^{w}$ values from such a survey, consider the diagram in Figure 10.

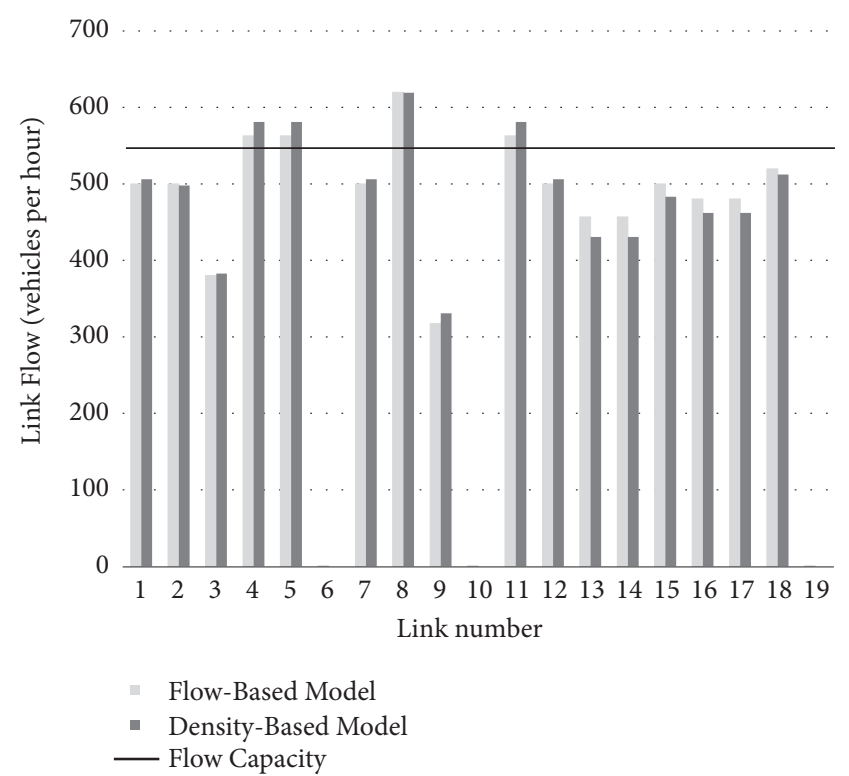

Figure 9: Link Flows, Both Approaches (low congestion).

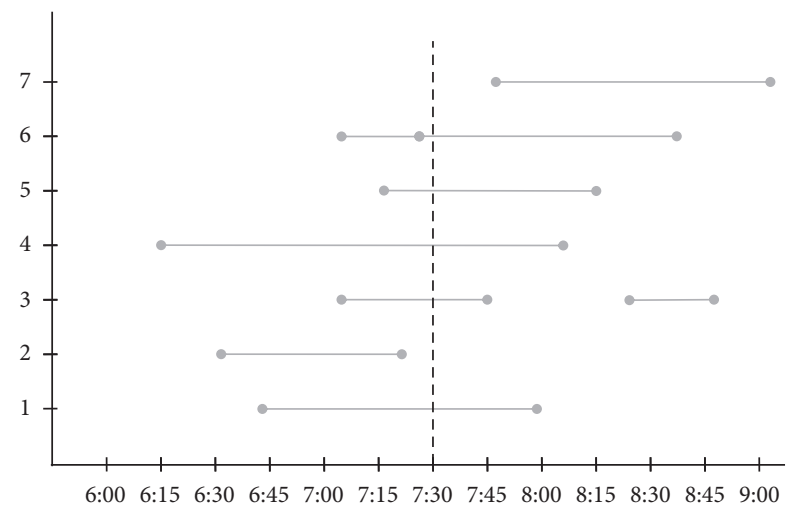

FIgURE 10: Trip Diagram from a Survey.

On the vertical axis are the 7 individuals surveyed after expanding the representative sample while on the horizontal axis is the time of day. As can be seen, Individual 1 departed at about $6: 45$ and arrived at 8:00. Individual 2 set out at 6:30 and arrived between 7:15 and 7:30. Individual 3 made two trips, the first one starting shortly after 7:00 and ending at 7:45 and the second one beginning soon after 8:15 and ending at about $8: 45$. By proceeding in this manner, the individuals or vehicles in the network at a given moment can be successively identified. For example, at 7:30 there were 6 individuals in the network. Since the survey also gathered the trip origins and destinations, an estimate of $N^{w}$ can then be obtained.

\section{Conclusions}

This paper developed, validated, and implemented a deterministic traffic assignment model based on link densities. The proposed formulation solves a variational inequality in a manner consistent with the relationship between flow, cost 
Table 6: Equilibrium Link Assignments, Density-Based Approach (low congestion).

\begin{tabular}{|c|c|c|c|c|c|c|c|}
\hline Link & $N_{a}$ & $l_{a}$ & $D_{a}$ & $D_{a}^{*}$ & $V_{a}$ & $c_{a}$ & $f_{a}$ \\
\hline 1 & 70.5055 & 5 & 14.1011 & 25 & 35.8989 & 0.1393 & 506.2138 \\
\hline 2 & 13.7323 & 1 & 13.7323 & 30 & 36.2677 & 0.0276 & 498.0380 \\
\hline 3 & 94.3981 & 10 & 9.4398 & 30 & 40.5602 & 0.2465 & 382.8805 \\
\hline 4 & 55.1230 & 3 & 18.3743 & 25 & 31.6257 & 0.0949 & 581.1005 \\
\hline 5 & 36.7487 & 2 & 18.3743 & 25 & 31.6257 & 0.0632 & 581.1005 \\
\hline 6 & 0.0000 & 9 & 0.0000 & 30 & 50.0000 & 0.1800 & 0.0000 \\
\hline 7 & 28.2022 & 2 & 14.1011 & 25 & 35.8989 & 0.0557 & 506.2138 \\
\hline 8 & 226.1471 & 10 & 22.6147 & 25 & 27.3853 & 0.3652 & 619.3104 \\
\hline 9 & 39.2293 & 5 & 7.8459 & 25 & 42.1541 & 0.1186 & 330.7354 \\
\hline 10 & 0.0000 & 10 & 0.0000 & 25 & 50.0000 & 0.2000 & 0.0000 \\
\hline 11 & 36.7487 & 2 & 18.3743 & 30 & 31.6257 & 0.0632 & 581.1005 \\
\hline 12 & 28.2022 & 2 & 14.1011 & 30 & 35.8989 & 0.0557 & 506.2138 \\
\hline 13 & 22.1236 & 2 & 11.0618 & 25 & 38.9382 & 0.0514 & 430.7267 \\
\hline 14 & 22.1236 & 2 & 11.0618 & 30 & 38.9382 & 0.0514 & 430.7267 \\
\hline 15 & 26.1893 & 2 & 13.0947 & 30 & 36.9053 & 0.0542 & 483.2632 \\
\hline 16 & 36.7122 & 3 & 12.2374 & 30 & 37.7626 & 0.0794 & 462.1156 \\
\hline 17 & 36.7122 & 3 & 12.2374 & 25 & 37.7626 & 0.0794 & 462.1156 \\
\hline 18 & 28.7754 & 2 & 14.3877 & 30 & 35.6123 & 0.0562 & 512.3795 \\
\hline 19 & 0.0000 & 2 & 0.0000 & 30 & 50.0000 & 0.0400 & 0.0000 \\
\hline
\end{tabular}

TABle 7: Equilibrium Link Assignments, Both Approaches (high congestion).

\begin{tabular}{|c|c|c|c|c|c|}
\hline \multirow{2}{*}{ Link } & \multirow{2}{*}{$\begin{array}{l}\text { Maximum flow for Density-Based model } \\
\qquad f_{a}^{\max }\end{array}$} & \multicolumn{2}{|c|}{ Flow-Based Model } & \multicolumn{2}{|c|}{ Density-Based Model } \\
\hline & & $c_{a}$ & $f_{a}$ & $c_{a}$ & $f_{a}$ \\
\hline 1 & 625 & 0.2002 & 663.2872 & 0.2025 & 624.9073 \\
\hline 2 & 625 & 0.0306 & 546.7128 & 0.0312 & 575.0042 \\
\hline 3 & 625 & 0.3090 & 551.9899 & 0.3662 & 619.6738 \\
\hline 4 & 625 & 0.1122 & 637.6172 & 0.1366 & 615.7452 \\
\hline 5 & 625 & 0.0748 & 637.6116 & 0.0636 & 583.4237 \\
\hline 6 & 625 & 0.1848 & 0.0028 & 0.1800 & 0.0000 \\
\hline 7 & 625 & 0.0801 & 663.2872 & 0.0810 & 624.9073 \\
\hline 8 & 625 & 0.4422 & 698.0101 & 0.5160 & 593.4209 \\
\hline 9 & 625 & 0.1332 & 461.0855 & 0.1498 & 554.7684 \\
\hline 10 & 625 & 0.2053 & 0.0056 & 0.2000 & 0.0000 \\
\hline 11 & 625 & 0.0748 & 637.6089 & 0.0633 & 581.1875 \\
\hline 12 & 625 & 0.0801 & 663.2899 & 0.0758 & 623.0643 \\
\hline 13 & 625 & 0.0643 & 572.2073 & 0.0542 & 482.7217 \\
\hline 14 & 625 & 0.0643 & 572.2129 & 0.0595 & 551.0790 \\
\hline 15 & 625 & 0.0678 & 596.6946 & 0.0646 & 589.3545 \\
\hline 16 & 625 & 0.0996 & 586.8883 & 0.0864 & 530.3997 \\
\hline 17 & 625 & 0.0996 & 586.8883 & 0.0864 & 530.3997 \\
\hline 18 & 625 & 0.0705 & 613.1272 & 0.0591 & 546.7634 \\
\hline 19 & 625 & 0.0411 & 0.0154 & 0.0400 & 0.0000 \\
\hline
\end{tabular}

(the inverse of speed), and density given by the fundamental traffic equation for each network link. The solution thus derived is a network traffic equilibrium of link densities that satisfies Wardrop's first principle.

The model's use of link densities to determine traffic equilibrium has a number of advantages over the traditional flow-based approach. Firstly, it recognizes that link capacity (or maximum flow) is not fixed but rather is a function of density levels. In other words, the maximum flow that can cross a link is variable and depends on demand, that is, density. Secondly, and, as a consequence of the foregoing, the proposed approach prevents flows from exceeding the links' theoretical maximum capacities. These maximum flows or capacities are determined as a function of the speed and 
TABle 8: Equilibrium Link Assignments, Both Approaches (low congestion).

\begin{tabular}{|c|c|c|c|c|c|}
\hline \multirow{2}{*}{ Link } & \multirow{2}{*}{$\begin{array}{l}\text { Maximum flow for Density-Based model } \\
\qquad f_{a}^{\max }\end{array}$} & \multicolumn{2}{|c|}{ Flow-Based Model } & \multicolumn{2}{|c|}{ Density-Based Model } \\
\hline & & $c_{a}$ & $f_{a}$ & $c_{a}$ & $f_{a}$ \\
\hline 1 & 625 & 0.1410 & 499.9880 & 0.1393 & 506.2138 \\
\hline 2 & 900 & 0.0282 & 500.0120 & 0.0276 & 498.0380 \\
\hline 3 & 900 & 0.2426 & 380.2809 & 0.2465 & 382.8805 \\
\hline 4 & 625 & 0.0947 & 562.9694 & 0.0949 & 581.1005 \\
\hline 5 & 625 & 0.0631 & 562.9647 & 0.0632 & 581.1005 \\
\hline 6 & 900 & 0.1848 & 0.0024 & 0.1800 & 0.0000 \\
\hline 7 & 625 & 0.0564 & 499.9880 & 0.0557 & 506.2138 \\
\hline 8 & 625 & 0.3579 & 619.7191 & 0.3652 & 619.3104 \\
\hline 9 & 625 & 0.1151 & 317.3234 & 0.1186 & 330.7354 \\
\hline 10 & 625 & 0.2053 & 0.0048 & 0.2000 & 0.0000 \\
\hline 11 & 900 & 0.0631 & 562.9623 & 0.0632 & 581.1005 \\
\hline 12 & 900 & 0.0564 & 499.9904 & 0.0557 & 506.2138 \\
\hline 13 & 625 & 0.0530 & 456.7820 & 0.0514 & 430.7267 \\
\hline 14 & 900 & 0.0530 & 456.7867 & 0.0514 & 430.7267 \\
\hline 15 & 900 & 0.0564 & 499.9982 & 0.0542 & 483.2632 \\
\hline 16 & 900 & 0.0821 & 480.2606 & 0.0794 & 462.1156 \\
\hline 17 & 625 & 0.0821 & 480.2606 & 0.0794 & 462.1156 \\
\hline 18 & 900 & 0.0583 & 519.7509 & 0.0562 & 512.3795 \\
\hline 19 & 900 & 0.0411 & 0.0115 & 0.0400 & 0.0000 \\
\hline
\end{tabular}

TABle 9: Costs and Flows Relative Difference, Both Approaches (two congestion scenarios).

\begin{tabular}{lcccc}
\hline Scenario & $\mathrm{N}^{\circ}$ of vehicles & Flows & Link Cost Relative distance & Link Flow Relative distance \\
\hline high congestion & $N_{1}=1237.4$ & $T_{1}=2460$ & 0.1382 & 0.0994 \\
low congestion & $N_{2}=801.67$ & $T_{2}=2000$ & 0.0219 & 0.0303 \\
\hline
\end{tabular}

density on each link as given by the fundamental traffic equation. Thirdly, the density-based model identifies whether a reduced flow level on a given link is due to low latent demand for its use (e.g., low density) or, on the contrary, to high congestion (e.g., high density) reducing the flow that can use the link, thereby generating traffic queues and longer delays.

An added benefit is that the estimates the model generates of link densities and their impacts on flow levels that can effectively use network links provide important data for planning road networks, calculating toll road revenues, and designing road pricing mechanisms based on traffic saturation levels.

The proposed model was validated using a traffic microsimulator (see Appendix A) and applied to a numerical example based on the well-known Nguyen-Dupuis network. It was then compared to the classical flow-based approach for a road system project involving the addition of a link. The results showed that flow-based models tend to underestimate network equilibrium cost and allow flows that often exceed the links' theoretical capacities even in low congestion scenarios. Furthermore, by underestimating costs the traditional approach also underestimates the net benefits of road system expansion projects or policies such as road pricing that lower vehicles use. These benefits arise from the reduction in the use of hypercongested networks attendant upon the implementation of a new link or a vehicle use disincentive policy, which as well as cutting costs increases road network capacity. The density-based approach, by contrast, takes these improvements into account.

In light of the above, we believe our proposed model based on densities constitutes a new and innovative methodology for analyzing and evaluating road and other transport projects and policies involving networks subject to congestion. The model's density-based approach overcomes a number of limitations inherent in the classical methodologies based on route flows.

A disadvantage is the difficulty in applying an algorithm whose solution satisfies the flow conservation property. A future work on this topic could consist of minimizing the relative error in flow conservation over the set of solutions of $\operatorname{VI}(C, K)$, which lead to a MPEC (Mathematical Programming with Equilibrium Constraints). The numerical resolution of MPECs has been studied by many authors (see, e.g., $[46-48])$.

\section{Appendix}

\section{A. Validation of the Proposed Model Using Microsimulations}

The validation of the density-based model was performed for a small road network, the same as the one developed in De 
$(3,350 \mathrm{~m})$

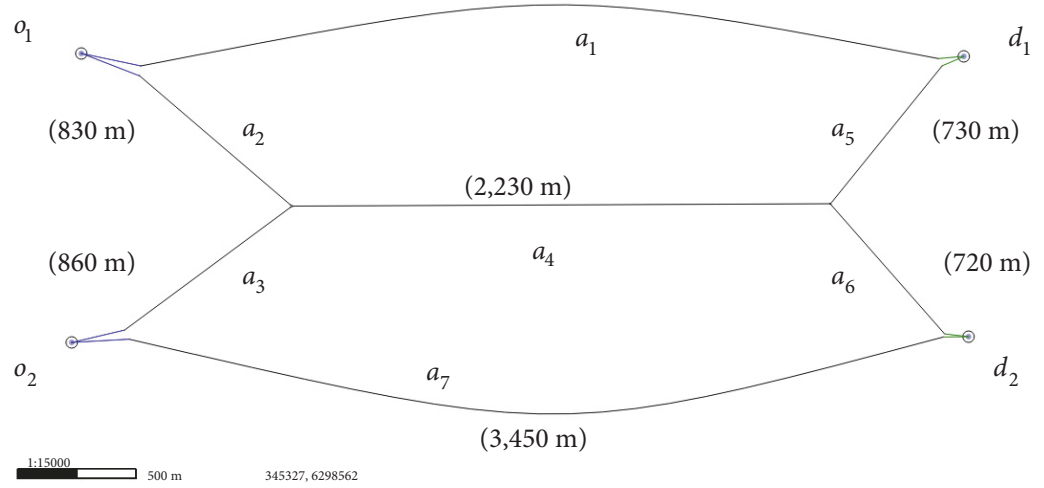

FIgURE 11: Road Network Microsimulated in Aimsun.

Grange et al. [49], using the Aimsun traffic microsimulator [50]. The network, described in Figure 11, is made up of 7 links, 6 nodes, and 2 O-D pairs. The link lengths are shown in the figure in parentheses.

The microsimulations were used to calibrate the relationship between speed and density, which we defined as $V_{a}\left(D_{a}\right)=\alpha-\beta D_{a}$, and to obtain input data for the model consisting of various values of $N^{w}$. The network was simulated for different levels of demand. For each such level, 10 one-hour replications were carried out, and speed and average density data were stored every 30 minutes for each link. The $\alpha$ and $\beta$ parameters were estimated using ordinary least squares to be $\widehat{\alpha}=76.34$ and $\widehat{\beta}=0.57$. A scatter graph of the dispersion between trip time (cost) and density is shown in Figure 12.

To generate the input data, we assumed two O-D pairs under different demand levels between pair $o_{1}-d_{1}$ and pair $o_{2}-d_{2}$. For each level, 30 one-hour replications were executed. The assignment of trips to the network using the microsimulator assumed a multinomial route-choice model with the following specification:

$$
h_{w}^{p}=T_{w} \frac{\exp \left(-\theta t_{w}^{p}\right)}{\sum_{p \in P^{w}} \exp \left(-\theta t_{w}^{p}\right)}, \quad \forall p, w
$$

where $t_{w}^{p}$ is the trip time (in hours) of route $p$ joining O-D pair $w$. Also, we assumed a high value for the time parameter $(\theta=200)$ and used 10-second interval between recalculations of the shortest route in order to force the microsimulator to obtain an assignment consistent with a Wardrop user equilibrium. The routes used are defined in Table 10.

Using the $N^{w}$ values obtained from the simulations and the parameter values derived above $(\widehat{\alpha}=76.34, \widehat{\beta}=0.57)$, we solved the variational inequality for the model to obtain the link flows $\left(f_{a}\right)$, number of vehicles on links $\left(N_{a}\right)$, link costs $\left(c_{a}\right)$, and O-D pair costs $\left(C_{w}\right)$. The results are shown in Figures 13-16. The averages for the traffic microsimulations are on the vertical axes of the figures while the corresponding values for the model are on the horizontal axes. The comparisons confirm that, for this validation example, the proposed

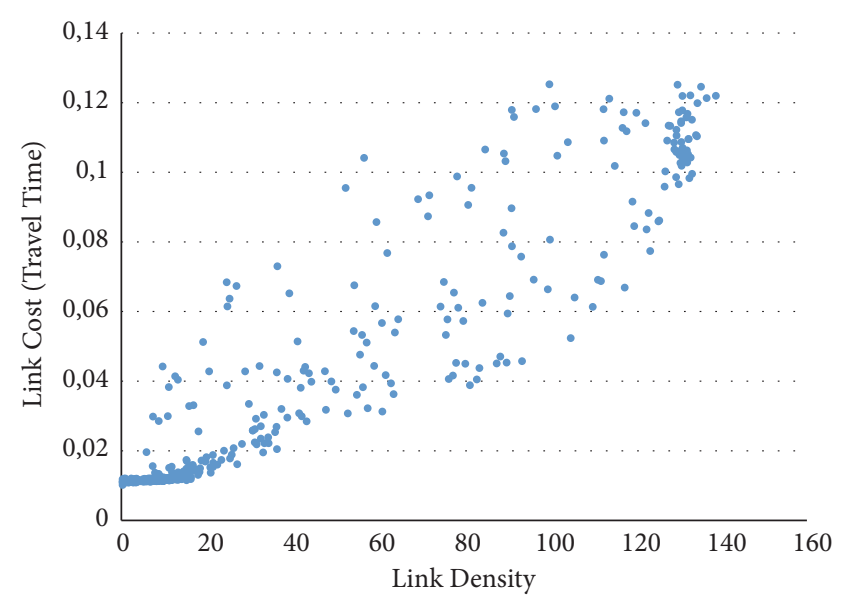

FIGURE 12: Microsimulation of Cost-Density Relationship.

TABLE 10: O-D Trips and Routes for Microsimulation.

\begin{tabular}{lc}
\hline O-D pair $w$ & Route link sequences \\
\hline$o_{1}-d_{1}$ & p1: $a_{1}$ \\
$o_{1}-d_{1}$ & p2: $a_{2}-a_{4}-a_{5}$ \\
$o_{2}-d_{2}$ & p3: $a_{3}-a_{4}-a_{6}$ \\
$o_{2}-d_{2}$ & p4: $a_{7}$ \\
\hline
\end{tabular}

model's results and those generated by the microsimulator are very similar.

Finally, the maximum gap function for flow-based model (considering all scenarios) was $9.2170 \cdot 10^{-4}$, and for flowbased model was $9.579 \cdot 10^{-6}$.

\section{B. General Example of a Road Network That Satisfies the Hypothesis behind the Solution Existence Theorem}

In what follows we develop a general example of a road network under congestion that satisfies the hypothesis (11) underlying the solution existence theorem proposed in the 


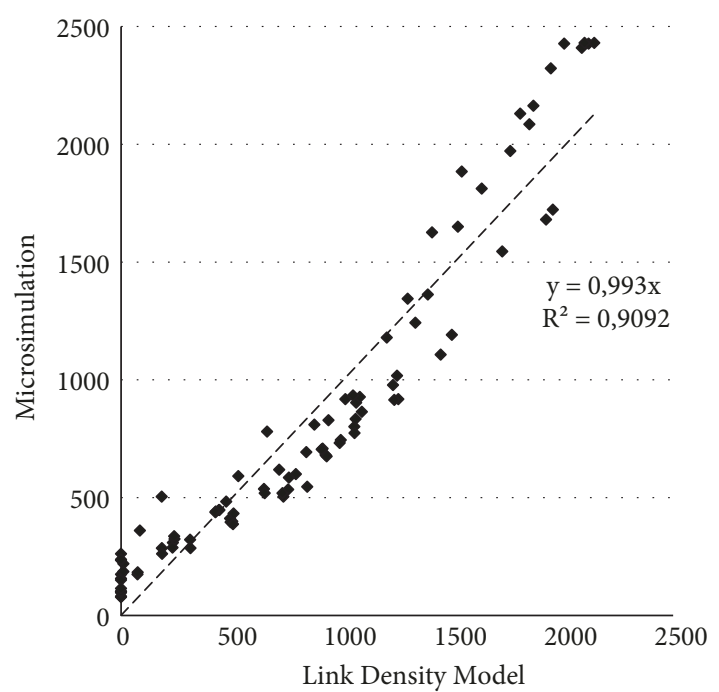

- fa

Figure 13: Link Flow Dispersion: Microsimulation vs DensityBased Model.

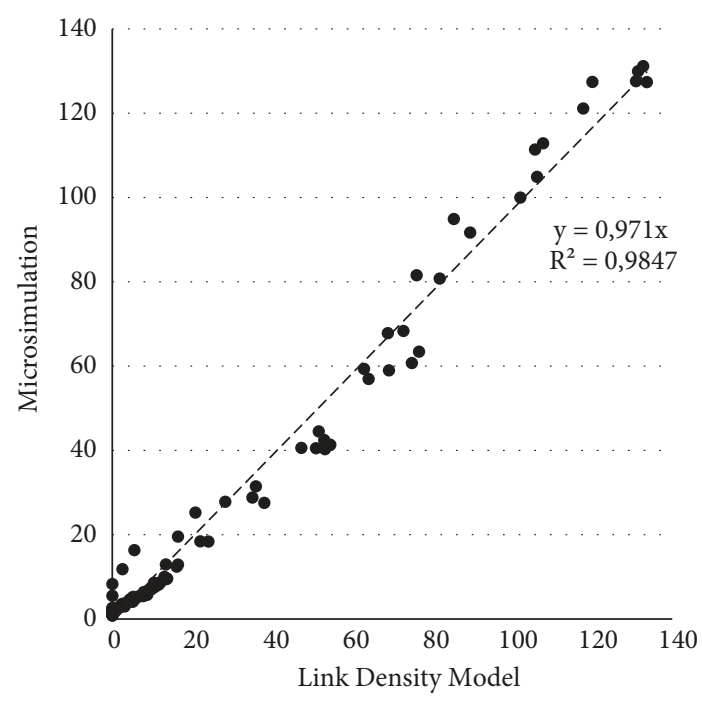

- $\mathrm{Na}$

FIGURE 14: Dispersion of No. of Vehicles on Links: Microsimulation vs Density-Based Model.

main text for the variational inequality used to obtain the Wardrop equilibrium based on link densities.

Proposition B.1 (assume that $K \neq \bar{K}$ ). Suppose that, for all $N^{*} \in \bar{K} \backslash K$ and all origin-destination pairs $w$, there exists a route $p \in P^{w}$ such that, for all links a that satisfy $\delta_{a p}=1$, we have $N_{a}^{*}<N_{a}^{\max }$. Then, for all $N^{*} \in \bar{K} \backslash K$, there exists an element $N \in K$ such that, for any sequence $N^{n} \in K$ which satisfies $\lim _{n \rightarrow \infty} N^{n}=N^{*}$, we have

$$
\lim _{n \longrightarrow \infty} C\left(N^{n}\right) \cdot\left(N-N^{n}\right)=-\infty
$$

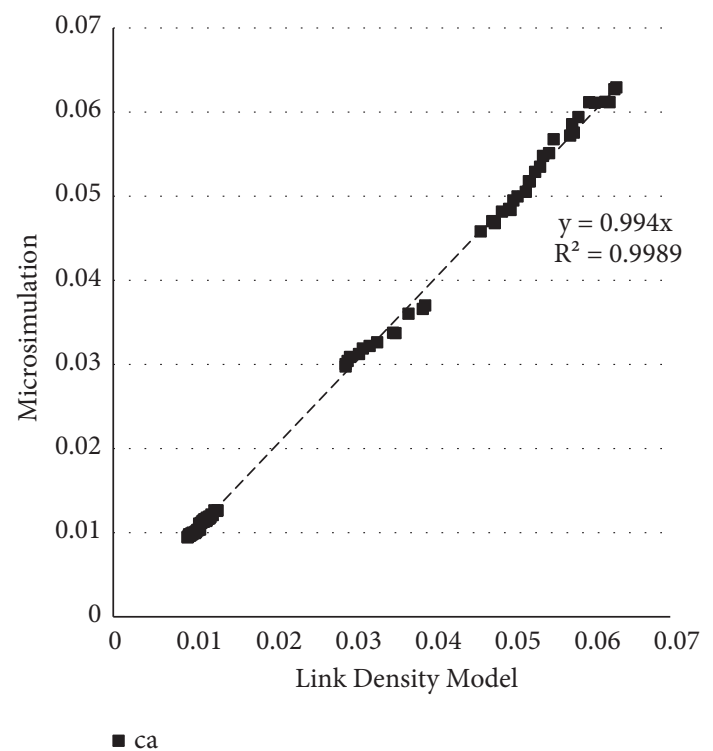

FIgURE 15: Link Cost Dispersion: Microsimulation vs Density-Based Model.

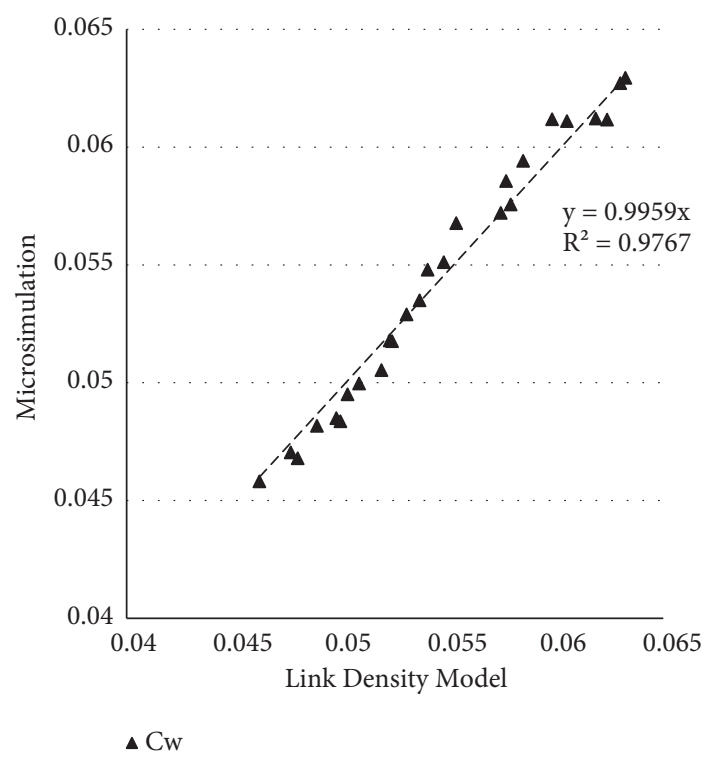

FIGURE 16: O-D Pair Dispersion: Microsimulation vs Density-Based Model.

That is, the condition that $\lim _{n \rightarrow \infty} C\left(N^{n}\right) .\left(N-N^{n}\right)<0$ is satisfied.

Proof (let $N^{*} \in \bar{K} \backslash K$ ). For each origin-destination pair $w$, we define $P_{\infty}^{w}=\left\{p \in P^{w} \mid \exists a \in L, \delta_{a p}=1, N_{a}^{*}=N_{a}^{\max }\right\}$ and $P_{0}^{w}=\left\{p \in P^{w} \mid \forall a \in L, \delta_{a p}=1, N_{a}^{*}<N_{a}^{\max }\right\}$. By hypothesis, $P_{0}^{w} \neq \varnothing$ for all origin-destination pairs $w$. Therefore, for each origin-destination pair $w$, we choose an element $p_{0}^{w} \in P_{0}^{w}$.

Given an $\varepsilon>0$, we construct a vector $N^{\varepsilon}=\left[N_{p}^{\varepsilon}\right]$ in the following manner. For each origin-destination pair $w$ and each $p \in P_{\infty}^{w}$, if $N_{p}^{*}>0$, then $N_{p}^{\varepsilon}=N_{p}^{*}-\varepsilon$, and if $N_{p}^{*}=0$, then $N_{p}^{\varepsilon}=0$. We also define $N_{p_{0}^{w}}^{\varepsilon}=N_{p_{0}^{w}}^{*}+k^{w} \varepsilon$, where $k^{w}$ is 
the cardinal of the set $\left\{p \in P_{\infty}^{w}, N_{p}^{*}>0\right\}$, and $N_{p}^{\varepsilon}=N_{p}^{*}$ if $p \in P_{0}^{w} \backslash\left\{p_{0}^{w}\right\}$. We will prove that if $\varepsilon>0$ is sufficiently small, then $N^{\varepsilon} \in K$.

First, we choose an $\varepsilon>0$ sufficiently small that, for all $p \in$ $P$, we have $N_{p}^{\varepsilon} \geq 0$. Observe that, for each origin-destination pair $w$,

$$
\begin{aligned}
\sum_{p \in P^{w}} N_{p}^{\varepsilon}= & \sum_{p \in P_{\infty}^{w}} N_{p}^{\varepsilon}+\sum_{p \in P_{0}^{w} \backslash\left\{p_{0}^{w}\right\}} N_{p}^{\varepsilon}+N_{p_{0}^{w}}^{\varepsilon} \\
\sum_{p \in P^{w}} N_{p}^{\varepsilon}= & \sum_{p \in P_{\infty}^{w}, N_{p}^{*}>0}\left(N_{p}^{*}-\varepsilon\right)+\sum_{p \in P_{0}^{w} \backslash\left\{p_{0}^{w}\right\}} N_{p}^{*}+N_{p_{0}^{w}}^{*} \\
& +k^{w} \varepsilon=\sum_{p \in P^{w}} N_{p}^{*}=N^{w}
\end{aligned}
$$

That is, with vector $N^{\varepsilon}=\left[N_{p}^{\varepsilon}\right]$ the restriction on the number of vehicles travelling between pair $w$ is satisfied.

Second, we show that no link's cost is infinite with vector $N^{\varepsilon}=\left[N_{p}^{\varepsilon}\right]$. Let link $a$ be such that $N_{a}^{*}=N_{a}^{\max }$. Then

$$
\begin{aligned}
N_{a}^{\varepsilon} & =\sum_{p \in P} q_{a p} N_{p}^{\varepsilon}=\sum_{w \in W} \sum_{p \in P_{\infty}^{w}} q_{a p} N_{p}^{\varepsilon} \\
& =\sum_{w \in W} \sum_{p \in P_{\infty}^{w}, N_{p}^{*}>0} q_{a p} N_{p}^{\varepsilon} \\
N_{a}^{\varepsilon} & =\sum_{w \in W} \sum_{p \in P_{\infty}^{w}, N_{p}^{*}>0} q_{a p}\left(N_{p}^{*}-\varepsilon\right) \\
& <\sum_{w \in W} \sum_{p \in P_{\infty}^{w}, N_{p}^{*}>0} q_{a p} N_{p}^{*}=N_{a}^{*}=N_{a}^{\max }
\end{aligned}
$$

The equality $\sum_{p \in P} q_{a p} N_{p}^{\varepsilon}=\sum_{w \in W} \sum_{p \in P_{\infty}^{w}} q_{a p} N_{p}^{\varepsilon}$ arises from the fact that, since $N_{a}^{*}=N_{a}^{\max }$, then $\left\{p \mid \delta_{a p}=\right.$ $1\} \subset \bigcup_{w} P_{\infty}^{w}$. Also, the equality $\sum_{w \in W} \sum_{p \in P_{\infty}^{w}} q_{a p} N_{p}^{\varepsilon}=$ $\sum_{w \in W} \sum_{p \in P_{\infty}^{w}, N_{p}^{*}>0} q_{a p} N_{p}^{\varepsilon}$ arises from the fact that, for each $w$, it is the case that $N_{p}^{*}=0 \Longrightarrow N_{p}^{\varepsilon}=0$ for all $p \in P_{\infty}^{w}$.

Now consider a link $a$ such that $N_{a}^{*}<N_{a}^{\max }$. We will prove that $N_{a}^{\varepsilon}<N_{a}^{\max }$ for all sufficiently small $\varepsilon>0$. To simplify the calculations, we will use the inequality $N_{p}^{\varepsilon} \leq N_{p}^{*}+k^{w} \varepsilon \leq$ $N_{p}^{*}+|P| \varepsilon$ for all routes $p$, where $|P|$ is the number of routes in the road network between all pairs $w$. We then have

$$
\begin{aligned}
N_{a}^{\varepsilon} & =\sum_{p \in P} q_{a p} N_{p}^{\varepsilon} \leq \sum_{p \in P} q_{a p}\left(N_{p}^{*}+|P| \varepsilon\right) \\
& =\sum_{p \in P} q_{a p} N_{p}^{*}+|P|^{2} \varepsilon=N_{a}^{*}+|P|^{2} \varepsilon
\end{aligned}
$$

Also, given that $N_{a}^{*}<N_{a}^{\max }$, if we choose a relatively small value of $\varepsilon$, then $N_{a}^{*}+|P|^{2} \varepsilon<N_{a}^{\max }$, which implies that $N_{a}^{\varepsilon}<$ $N_{a}^{\max }$. We therefore choose a sufficiently small $\varepsilon>0$ such that $N_{p}^{\varepsilon} \geq 0$ for all routes $p$ and $N_{a}^{\varepsilon}<N_{a}^{\max }$ for all links $a$. Thus, $N^{\varepsilon} \in K$.

For greater clarity, in what follows we denote the vector $N^{\varepsilon} \in K$ obtained above as $N$.
Given a sequence $\lim _{n \rightarrow \infty} N^{n}=N^{*}$ with $N^{n} \in K$, then

$$
\begin{aligned}
C & \left(N^{n}\right) \cdot\left(N-N^{n}\right)=\sum_{p \in P} C_{p}\left(N^{n}\right) \cdot\left(N_{p}-N_{p}^{n}\right) \\
& =\sum_{w \in W} \sum_{p \in P^{w}} C_{p}\left(N^{n}\right) \cdot\left(N_{p}-N_{p}^{n}\right) \\
& =\sum_{w \in W}\left(\sum_{p \in P_{0}^{w}} C_{p}\left(N^{n}\right) \cdot\left(N_{p}-N_{p}^{n}\right)\right. \\
& \left.+\sum_{p \in P_{\infty}^{w}} C_{p}\left(N^{n}\right) \cdot\left(N_{p}-N_{p}^{n}\right)\right) \\
& \leq \sum_{w \in W} \sum_{p \in P_{0}^{w}} C_{p}\left(N^{n}\right) \cdot\left(N_{p}-N_{p}^{n}\right) \\
& +\sum_{w \in W} \sum_{p \in P_{\infty}^{w}, N_{p}^{*}>0} C_{p}\left(N^{n}\right) \cdot\left(N_{p}-N_{p}^{n}\right) \\
& =\sum_{w \in W} \sum_{p \in P_{0}^{w}} C_{p}\left(N^{n}\right) \cdot\left(N_{p}-N_{p}^{n}\right) \\
& +\sum_{w \in W} \sum_{p \in P_{\infty}^{w}, N_{p}^{*}>0} C_{p}\left(N^{n}\right) \cdot\left(N_{p}^{*}-\varepsilon-N_{p}^{n}\right)
\end{aligned}
$$

The inequality in the third line of (B.5) is the consequence of the fact that if $p \in P_{\infty}^{w}$ and $N_{p}^{*}=0$, then, by construction, $N_{p}=0$ and, therefore, $\sum_{p \in P_{\infty}^{w}, N_{p}^{*}=0} C_{p}\left(N^{n}\right) \cdot\left(N_{p}-N_{p}^{n}\right)=$ $\sum_{p \in P_{\infty}^{w}, N_{p}^{*}=0} C_{p}\left(N^{n}\right) \cdot\left(-N_{p}^{n}\right) \leq 0$. Note that, in the last line of (B.5), we use the equality $N_{p}=N_{p}^{*}-\varepsilon$ when $p \in P_{\infty}^{w}$ and $N_{p}^{*}>0$.

The quantity $\sum_{w \in W} \sum_{p \in P_{0}^{\omega}} C_{p}\left(N^{n}\right) .\left(N_{p}-N_{p}^{n}\right)$ is bounded when $n \longrightarrow+\infty$ given that $\lim _{n \rightarrow \infty} C_{p}\left(N^{n}\right)=$ $\sum_{a \in L} C_{a}\left(N_{a}^{*}\right) \delta_{a p}<+\infty$ if $p \in P_{0}^{w}$. Also, $\lim _{n \rightarrow \infty}\left(N_{p}^{*}-\varepsilon-\right.$ $\left.N_{p}^{n}\right)=-\varepsilon<0$ and $\lim _{n \rightarrow \infty} C_{p}\left(N^{n}\right)=+\infty$ if $p \in P_{\infty}^{w}$, the latter implying further that $\lim _{n \rightarrow \infty} C_{p}\left(N^{n}\right)\left(N_{p}-N_{p}^{n}\right)=-\infty$.

Thus, we have demonstrated $\lim _{n \rightarrow \infty} C\left(N^{n}\right) \cdot\left(N-N^{n}\right)=$ $-\infty$.

\section{System Optimal Assignment Model Based in Link Densities}

The optimization problem that gives the system's optimal equilibrium assignment is as follows:

$$
\begin{array}{ll}
\min _{\left\{N_{p}\right\}} & \sum_{a \in L} c_{a}\left(N_{a}\right) \cdot N_{a} \\
\text { s.t.: } & N_{a}=\sum_{p \in P} N_{p} q_{a p} \quad \forall a \in L
\end{array}
$$




$$
\begin{aligned}
& N^{w}=\sum_{p \in P^{w}} N_{p} \quad \forall w \in W \\
& N_{p} \geq 0 \quad \forall p \in P \\
& N_{a}<N_{a}^{\max }
\end{aligned}
$$

where $N_{a}^{\max }$ is the maximum number of vehicles that can be present on link $a$. Since the set of constraints in (C.1) is not closed, consider the following version:

$$
\begin{array}{ll}
\min _{\left\{N_{p}\right\}} \quad \sum_{a \in L} c_{a}\left(N_{a}\right) \cdot N_{a} \\
\text { s.t.: } \quad N_{a}=\sum_{p \in P} N_{p} q_{a p} \quad \forall a \in L \\
N^{w}=\sum_{p \in P^{w}} N_{p} \quad \forall w \in W \\
N_{p} \geq 0 \quad \forall p \in P \\
N_{a} \leq N_{a}^{\max }-\varepsilon
\end{array}
$$

Observe that, in this version, the constraint set is compact and the objective function is continuous over the set, thus guaranteeing that solutions exist.

We now prove that the constraint $N_{a} \leq N_{a}^{\max }-\varepsilon$ is not active when $\varepsilon$ is sufficiently small.

Proposition C.1. There exists a number $\varepsilon_{0}>0$ such that, for all $\varepsilon \in] 0, \varepsilon_{0}\left[\right.$, the solution of problem (6) satisfies $N_{a}<N_{a}^{\max }-\varepsilon$ for all links $a$.

Proof. Let $N=\left(N_{p}\right)_{p}$ be a vector such that $N_{a}<N_{a}^{\max }$ for all links $a$ and also let $=\sum_{a \in L} c_{a}\left(N_{a}\right) \cdot N_{a}$. Since $\lim _{N_{a} \longrightarrow N_{a}^{\max } c_{a}}\left(N_{a}\right) \cdot N_{a}=+\infty$, there exists an $\varepsilon_{0}>0$ such that, for every link $a, c_{a}\left(N_{a}\right) \cdot N_{a}>M$ for all $N_{a} \in\left[N_{a}^{\max }-\right.$ $\varepsilon_{0}, N_{a}^{\max }[$. Now let $\varepsilon \in] 0, \varepsilon_{0}\left[\right.$ and assume that $\bar{N}=\left(\bar{N}_{p}\right)_{p}$ is a solution of (6). We then prove that $\bar{N}_{a}<\bar{N}_{a}^{\max }-\varepsilon$ for all links $a$. Suppose that there exists a link $a$ such that $\bar{N}_{a}=\bar{N}_{a}^{\max }-\varepsilon$. Since $\varepsilon<\varepsilon_{0}$, we have $\bar{N}_{a}>N_{a}^{\max }-\varepsilon_{0}$ and therefore $\sum_{a \in L} c_{a}\left(\bar{N}_{a}\right) \cdot \bar{N}_{a}>M=\sum_{a \in L} c_{a}\left(N_{a}\right) \cdot N_{a}$. This contradicts the assumption that $\bar{N}=\left(\bar{N}_{p}\right)_{p}$ is a solution of (6). Therefore $\bar{N}_{a}<\bar{N}_{a}^{\max }-\varepsilon$ for all links $a$.

Having thus proved that, for all sufficiently small $\varepsilon>0$, the $N_{a} \leq N_{a}^{\max }-\varepsilon$ constraint is not active, the optimality conditions are

$$
\begin{aligned}
& \frac{\partial L}{\partial N_{p}}=\sum_{a \in L}\left(c_{a} \frac{\partial N_{a}}{\partial N_{p}}+\frac{\partial c_{a}}{\partial N_{a}} \frac{\partial N_{a}}{\partial N_{p}} N_{a}\right)-\lambda^{w}=0 \\
& \frac{\partial L}{\partial N_{p}}=\sum_{a \in L} q_{a p}\left(c_{a}+\frac{\partial c_{a}}{\partial N_{a}} N_{a}\right)-\lambda^{w}=0 \\
& \sum_{a \in L} q_{a p} \underbrace{\left(c_{a}+\frac{\partial c_{a}}{\partial N_{a}} N_{a}\right)}_{\text {marginal cost }}=\lambda^{w}
\end{aligned}
$$

Therefore, the equilibrium condition for the optimal assignment problem under a density-based approach is that, for each route used, the marginal cost of each link duly weighted by its relative length in the route and summed over the latter must be the same.

\section{Data Availability}

The exercises and numerical analyses that support the results presented in this article are based on large amounts of data. The reader can personally contact the authors if these data are required.

\section{Disclosure}

The research presented corresponds to the academic work carried out by the authors within their academic commitment at the School of Industrial Engineering of the Universidad Diego Portales. No additional resources were used.

\section{Conflicts of Interest}

The authors declare that there are no conflicts of interest regarding the publication of this paper.

\section{References}

[1] J. G. Wardrop, "Some theoretical aspects of road traffic research," Proceedings Institution of Civil Engineers, vol. 1, no. 3, pp. 325-362, 1952.

[2] F. H. Knight, "Some fallacies in the interpretation of social cost," The Quarterly Journal of Economics, vol. 38, no. 4, pp. 582-606, 1924.

[3] M. J. Beckmann, C. B. McGuire, and C. B. Winsten, Studies in the Economics of Transportation, Yale University Press, New Haven, Conn, USA, 1956.

[4] S. C. Dafermos and F. T. Sparrow, "The traffic assignment problem for a general network," Journal of Research of the National Bureau of Standards, vol. 73B, pp. 91-118, 1969.

[5] M. J. Smith, "The existence, uniqueness and stability of traffic equilibria," Transportation Research Part B: Methodological, vol. 13, no. 4, pp. 295-304, 1979.

[6] S. Dafermos, "Traffic equilibrium and variational inequalities," Transportation Science, vol. 14, no. 1, pp. 42-54, 1980.

[7] S. Dafermos, "Relaxation algorithms for the general asymmetric traffic equilibrium problem," Transportation Science, vol. 16, no. 2, pp. 231-240, 1982.

[8] M. Florian and H. Spiess, "The convergence of diagonalization algorithms for asymmetric network equilibrium problems," Transportation Research Part B: Methodological, vol. 16, no. 6, pp. 477-483, 1982.

[9] C. Fisk and S. Nguyen, "Solution algorithms for network equilibrium models with asymmetric user costs," Transportation Science, vol. 16, no. 3, pp. 361-381, 1982.

[10] C. S. Fisk and D. E. Boyce, "Alternative variational inequality formulations of the equilibrium travel choice problem," Transportation Science, vol. 17, no. 4, pp. 454-463, 1983.

[11] A. B. Nagurney, "Comparative tests of multimodal traffic equilibrium methods," Transportation Research Part B: Methodological, vol. 18B, no. 6, pp. 469-485, 1984. 
[12] J. Hammond, Solving Asymmetric Variational Inequality Problems and Systems of Equations with Generalized Nonlinear Programming Algorithms [Ph.D. thesis], Department of Mathematics, MIT, Cambridge, Mass, USA, 1984.

[13] P. Marcotte and J. GuElat, "Adaptation of a modified Newton method for solving the asymmetric traffic equilibrium problem," Transportation Science, vol. 22, no. 2, pp. 112-124, 1988.

[14] G. Auchmuty, "Variational principles for variational inequalities," Numerical Functional Analysis and Optimization, vol. 10, no. 9-10, pp. 863-874, 1989.

[15] S. A. Gabriel and D. Bernstein, "The traffic equilibrium problem with nonadditive path costs," Transportation Science, vol. 31, no. 4, pp. 337-348, 1997.

[16] M. Patriksson, Nonlinear Programming and Variational Inequality Problems: A Unified Approach, vol. 23 of Applied Optimization, Kluwer Academic, Dordrecht, The Netherlands, 1998.

[17] C. F. Daganzo and Y. Sheffi, "On stochastic models of traffic assignment," Transportation Science, vol. 11, no. 3, pp. 253-274, 1977.

[18] M. L. Hazelton, "Some remarks on stochastic user equilibrium," Transportation Research Part B: Methodological, vol. 33, no. 2, pp. 101-108, 1998.

[19] M. S. Ramming, Network knowledge and route choice [unpublished dissertation], Massachusetts Institute of Technology, Cambridge, Mass, USA, 2001.

[20] J. N. Prashker and S. Bekhor, "Route choice models used in the stochastic user equilibrium problem: a review," Transport Reviews, vol. 24, no. 4, pp. 437-463, 2004.

[21] A. Karoonsoontawong and D.-Y. Lin, "Combined gravity model trip distribution and paired combinatorial logit stochastic user equilibrium problem," Networks and Spatial Economics, vol. 15, no. 4, pp. 1011-1048, 2015.

[22] M. Li and H. Huang, "A regret theory-based route choice model," Transportmetrica A: Transportation Science, vol. 13, no. 3, pp. 250-272, 2017.

[23] S. F. Batista, C. Zhao, and L. Leclercq, "Effects of users' bounded rationality on a traffic network performance: a simulation study," Journal of Advanced Transportation, vol. 2018, Article ID 9876598, 20 pages, 2018.

[24] Y. Lim and H. Kim, "A combined model of trip distribution and route choice problem," Transportmetrica A: Transport Science, vol. 12, no. 8, pp. 721-735, 2016.

[25] D. P. Watling, T. K. Rasmussen, C. G. Prato, and O. A. Nielsen, "Stochastic user equilibrium with a bounded choice model," Transportation Research Part B: Methodological, vol. 114, pp. 254-280, 2018.

[26] L. Zhao, H. Guan, J. Zhu, and Y. Wei, "A reliability-based network equilibrium model with adaptive risk-averse travelers," Journal of Advanced Transportation, vol. 2018, Article ID 5294185, 13 pages, 2018.

[27] E. T. Mtoi and R. Moses, "Calibration and evaluation of link congestion functions," Journal of Transportation Technologies, vol. 4, pp. 141-149, 2014.

[28] T. Larsson and M. Patriksson, "An augmented lagrangean dual algorithm for link capacity constrained traffic assignment problems," Transportation Research Part B, vol. 29, pp. 433-455, 1995.

[29] P. Ferrari, "Capacity constraints in urban transport networks," Transportation Research Part B: Methodological, vol. 31, no. 4, pp. 291-301, 1997.
[30] Y. Nie, H. M. Zhang, and D.-H. Lee, "Models and algorithms for the traffic assignment problem with link capacity constraints," Transportation Research Part B: Methodological, vol. 38, no. 4, pp. 285-312, 2004.

[31] B. Ran and D. Boyce, Modeling Dynamic Transportation Networks, Springer, Berlin, Germany, 1996.

[32] S. Peeta and A. K. Ziliaskopoulos, "Foundations of dynamic traffic assignment: the past, the present and the future," Networks and Spatial Economics, vol. 1, pp. 233-265, 2001.

[33] C. Tampere and F. Viti, "Dynamic traffic assignment under equilibrium and non-equilibrium: do we need a paradigm shift?" in Proceedings of the 3rd International Symposium on Dynamic Traffic Assignment, 2010.

[34] D. Boyce, D.-H. Lee, and B. Ran, "Analytical models of the dynamic traffic assignment problem," Networks and Spatial Economics, vol. 1, no. 3-4, pp. 377-390, 2001.

[35] W. Y. Szeto and H. K. Lo, "Dynamic traffic assignment: properties and extensions," Transportmetrica, vol. 2, no. 1, pp. 31-52, 2006.

[36] P. Liu, F. Liao, H.-J. Huang, and H. Timmermans, "Dynamic activity-travel assignment in multi-state supernetworks under transport and location capacity constraints," Transportmetrica A: Transport Science, vol. 12, no. 7, pp. 572-590, 2016.

[37] S. Calvert, M. Minderhoud, H. Taale, I. Wilmink, and V. L. Knoop, Traffic assignment and simulation models: State-of-theArt Background Document, Centre for Expertise on Traffic Management, Delft, Netherlands, 2016.

[38] V. T. Arasan and K. Krishnamurthy, "Effect of traffic volume on PCU of vehicles under heterogeneous traffic conditions," Road \& Transport Research, vol. 17, no. 1, pp. 32-49, 2008.

[39] H. Wang, J. Li, Q. Y. Chen, and D. Ni, "Speed-density relationship: from deterministic to stochastic," in Proceedings of the Transportation Research Board 88th Annual Meeting, vol. 10, 2009.

[40] R. Kucharski and A. Drabicki, "Estimating macroscopic volume delay functions with the traffic density derived from measured speeds and flows," Journal of Advanced Transportation, vol. 2017, Article ID 4629792, 10 pages, 2017.

[41] M. Martínez-Díaz and I. Pérez, "A simple algorithm for the estimation of road traffic space mean speeds from data available to most management centres," Transportation Research Part B: Methodological, vol. 75, pp. 19-35, 2015.

[42] L. De Grange, R. Troncoso, and F. González, "A road pricing model for congested highways based on link densities," Journal of Advanced Transportation, vol. 2017, Article ID 3127398, 12 pages, 2017.

[43] P. T. Harker and J.-S. Pang, "Finite-dimensional variational inequality and nonlinear complementarity problems: a survey of theory, algorithms and applications," Mathematical Programming, vol. 48, no. 1-3, pp. 161-220, 1990.

[44] S. Nguyen and C. Dupuis, "An efficient method for computing traffic equilibria in networks with asymmetric transportation costs," Transportation Science, vol. 18, no. 2, pp. 185-202, 1984.

[45] T. Wang, R. D. C. Monteiro, and J.-S. Pang, "An interior point potential reduction method for constrained equations," Mathematical Programming, vol. 74, no. 2, pp. 159-195, 1996.

[46] D. Ralph and S. J. Wright, "Some properties of regularization and penalization schemes for MPECs," Optimization Methods \& Software, vol. 19, no. 5, pp. 527-556, 2004.

[47] S. Scholtes, "Convergence properties of a regularization scheme for mathematical programs with complementarity constraints," SIAM Journal on Optimization, vol. 11, no. 4, pp. 918-936, 2001. 
[48] T. Hoheisel, C. Kanzow, and A. Schwartz, "Theoretical and numerical comparison of relaxation methods for mathematical programs with complementarity constraints," Mathematical Programming, vol. 137, no. 1-2, pp. 257-288, 2013.

[49] L. de Grange, F. Gonzalez, and S. Bekhor, "Path flow and trip matrix estimation using link flow density," Networks and Spatial Economics, vol. 17, no. 1, pp. 173-195, 2017.

[50] J. Casas, J. L. Ferrer, D. Garcia, J. Perarnau, and A. Torday, "Traffic simulation with aimsun," in Fundamentals of Traffic Simulation, pp. 173-232, Springer, New York, NY, USA, 2010. 


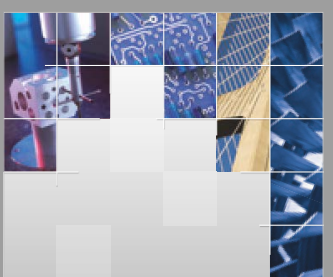

\section{Enfincering}
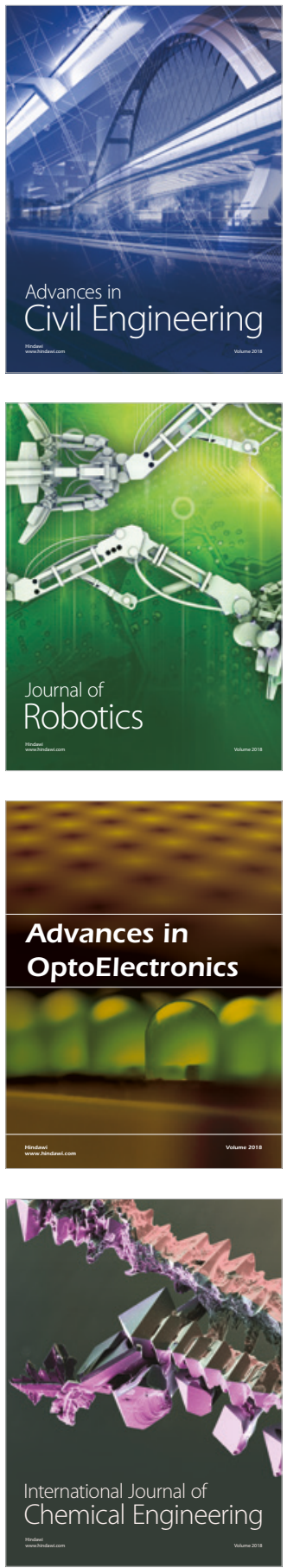

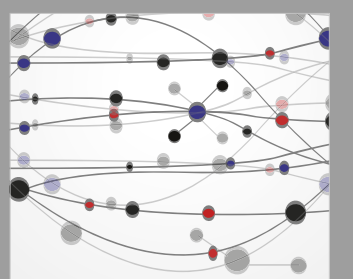

\section{Rotating \\ Machinery}

The Scientific World Journal

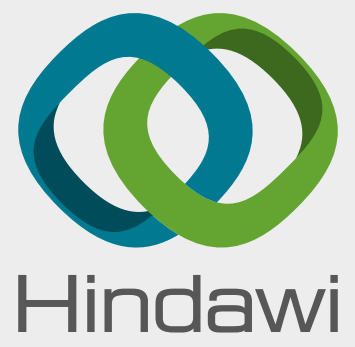

Submit your manuscripts at

www.hindawi.com
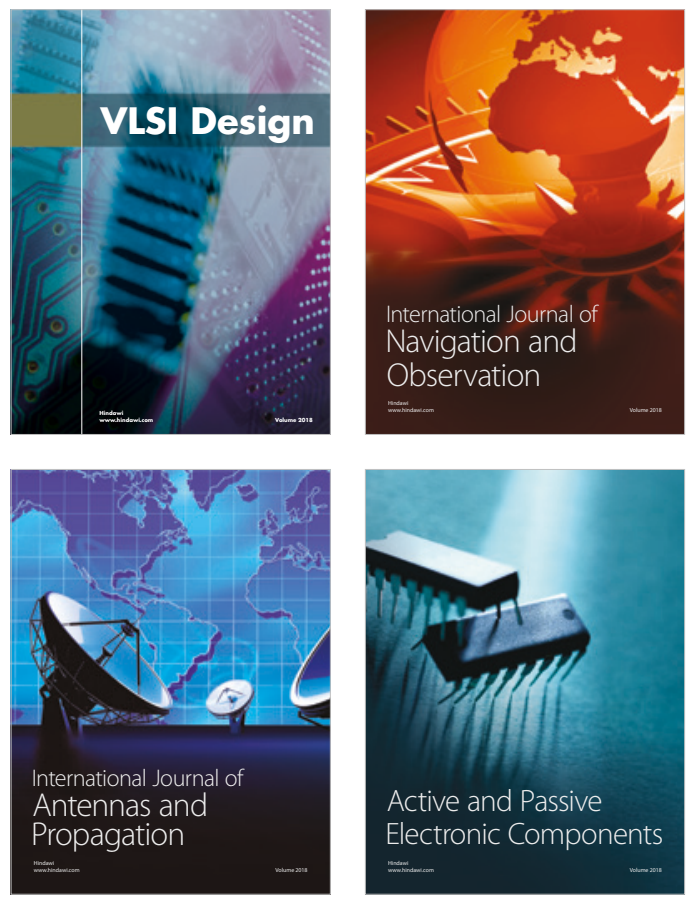
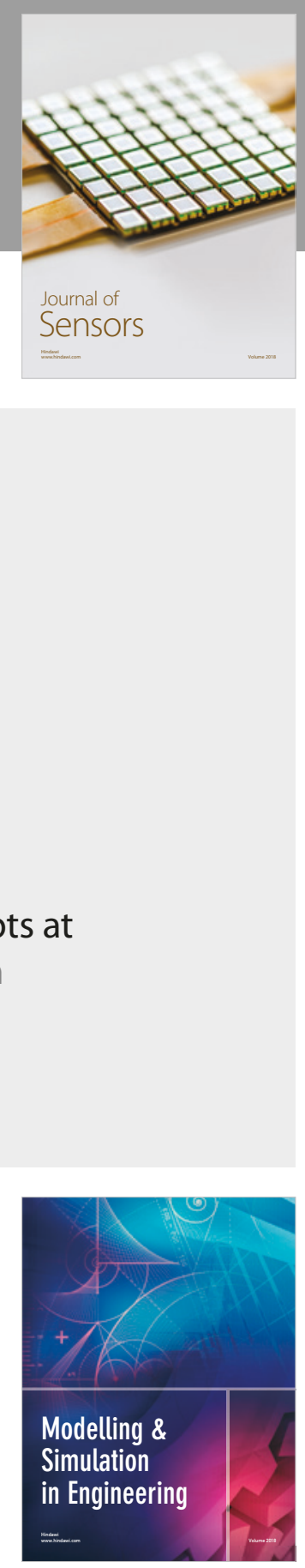

\section{Advances \\ Multimedia}
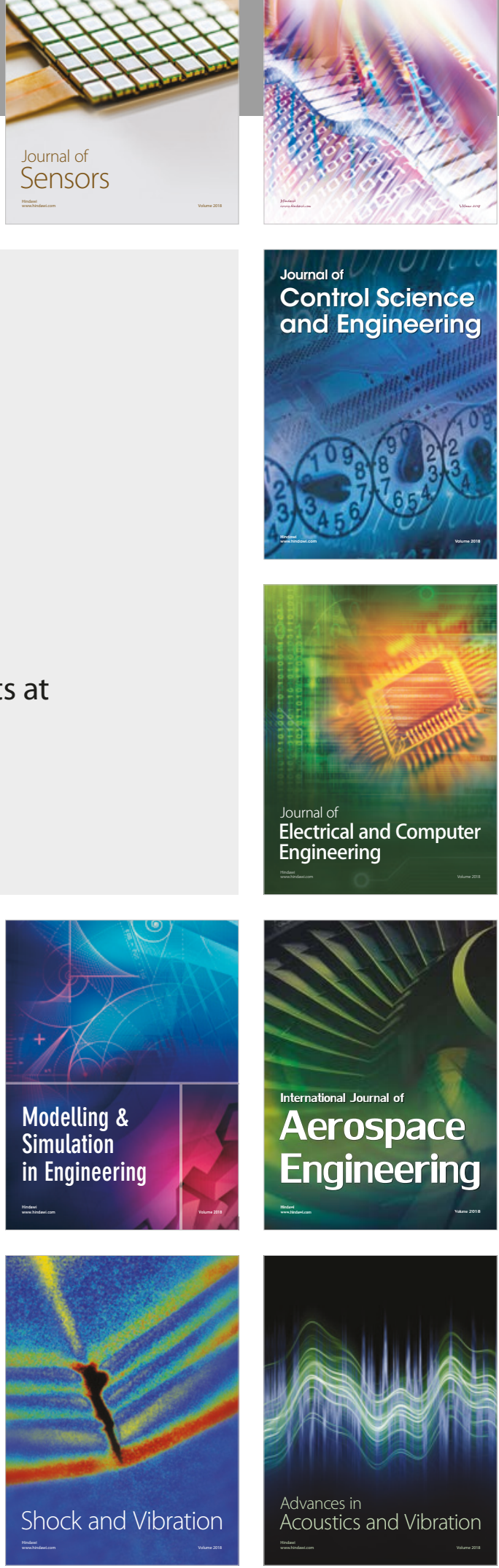